\title{
An Accurate Interest Matching Algorithm based on Prediction of the Space-Time Intersection of Regions for the Distributed Virtual Environment
}

\author{
Yong Peng, Mei Yang, Quanjun Yin ${ }^{1}$, Yabing Zha \\ College of Information Systems and Management, National University of Defense Technology, \\ Changsha, Hunan, P.R. China, 410073
}

\begin{abstract}
Interest matching is an important data-filtering mechanism for a large-scale distributed virtual environment. Many of the existing algorithms perform interest matching at discrete timesteps. Thus, they may suffer the missing-event problem: failing to report the events between two consecutive timesteps. Some algorithms solve this problem, by setting short timesteps, but they have a low computing efficiency. Additionally, these algorithms cannot capture all events, and some spurious events may also be reported. In this paper, we present an accurate interest matching algorithm called the predictive interest matching algorithm, which is able to capture the missing events between discrete timesteps. The PIM algorithm exploits the polynomial functions to model the movements of virtual entities, and predict the time intervals of region overlaps associated with the entities accurately. Based on the prediction of the space-time intersection of regions, our algorithm can capture all missing events and does not report the spurious events at the same time. To improve the runtime performance, a technique called region pruning is proposed and used in our algorithm. In experiments, we compare the new algorithm with the frequent interest matching algorithm and the space-time interest matching algorithm on the HLA/RTI distributed infrastructure. The results prove that although an additional matching effort is required in the new algorithm, it outperforms the baselines in terms of event-capturing ability, redundant matching avoidance, runtime efficiency and scalability.
\end{abstract}

Keywords: Interest management; Interest matching; Distributed simulation; DVE; HLA;

\section{Introduction}

A Distributed Virtual Environment (DVE) provides a virtual world where the entities can interact with each other. With the growing scale and complexity of the simulation system, the number of virtual entities in the virtual world may be extremely large. And all-to-all data exchange between computational nodes, on which virtual entities are hosted, will consume a large amount of network bandwidth and decrease the runtime performance of the system significantly. Therefore, efficient data distribution becomes crucial to reduce the exchange of irrelevant data between nodes and to meet performance and usability requirements [1-2].

In past decades, a number of techniques are proposed with the idea of interest management: they ensure the entities receive only interested data and filter the uninterested data. In the interest management mechanism, two regions in the virtual space are usually defined for each entity: the update region and the subscription region [3-5]. The update region is the area corresponds to the location of an entity and in which the entity is expected to be moving. The subscription region, which is also called area-of-interest (AOI), is the region in which the entity is interested. A subscription entity only needs to receive attribute updates from the other entities whose update regions overlap with subscription regions. The process of checking the overlap state of

${ }^{1}$ Corresponding author. Tel.: +86 731 84574590; E-mail address: yin_quanjun@ 163.com 
update-subscription region pairs is referred to Interest Matching (IM) [2].

Most of distributed simulations have the concept of logical time (simulation time). In real-time simulation, logical time is equal to physical time. In HLA, the logical time is represented by a variable. Time step in this paper has different meanings under different contexts. For advance of simulation time, a time step is one time of logical time advancement, which is not necessary in uniform step. For region update, a time step is an update of region. For IM algorithm, a time step is an execution of IM algorithm, which is a variable and is denoted as the beginning logical time of an execution. Generally, each time of IM algorithm execution is driven by the region update or by the advance of the simulation time.

Because entities update their update/subscription regions at discrete timesteps, most existing IM algorithms also perform matching processes at discrete timesteps. If those regions had been moving continuously from their locations at one time step to their locations at the next timestep, the discrete IM algorithms that cannot detect this overlap state will cause the missing-event happening. This may lead to the missing-event problem [6]: the IM algorithms can not capture all the actual events. Moreover, the separation of two overlapped regions is important but is ignored by many existing IM algorithms. For instance, in a military simulation, when an aircraft is flighting out of the range of a radar, a separating event occurs. If IM algorithms can report the accurate time of the separating event, the radar can make better decisions in the next step, and the aircraft can immediately stop updating attributes of the radar.

To filter the irrelevant messages accurately, some of the existing IM algorithms define multidimensional and complex update or subscription regions for virtual entities. However, matching these kinds of regions is always time consuming. Furthermore, because the update region and subscription region are changed frequently, many of the existing IM algorithms have to perform the region matching operations at every timestep [7-12], which produces many unnecessary overlap tests. Both of the two reasons make these IM algorithms may suffer heavy computational workload, which is unacceptable in some applications. Therefore, people always have to make a trade-off between filtering precision and runtime efficiency in the interest matching processes.

To improve the filter precision and the runtime efficiency in the IM processes simultaneously, we propose an accurate IM algorithm called the Predictive Interest Matching algorithm (PIM), which is based on the prediction of the space-time intersection of regions. The PIM algorithm uses multidimensional rectangles to represent the update and subscription regions of virtual entities, which is similar to the definition of the extent of a region in the routing space of HLA [13-14]. The space-time intersection of regions in our algorithm is predicted by solving the movement inequations of regions. By accurately predicting time intervals at which the update regions and the subscription regions overlap with each other, our algorithm can catch the missing events and distinguish the overlapping events of regions and the separating events of regions. Furthermore, by predicting the space-time intersection of regions, the PIM can avoid performing redundant interest matching operations.

To further improve the runtime efficiency of PIM, we introduce a region pruning method in the PIM, which is based on the maximum area of potential overlap of a region. The region pruning method is used for culling out the region pairs that are unlikely to overlap with each other. Our algorithm uses the region pruning method to test the potential overlap state of region pairs before predicting space-time intersections of the region pairs. In this way, many unnecessary overlap tests 
of regions can be avoided and the computational workload of the PIM algorithm is reduced.

In experiments, we test our algorithm in a World War II dogfight scenario, which is run on the HLAIRTI distributed simulation infrastructure. Four sets of experiments are conducted to evaluate event-capturing ability, redundant matching avoidance, runtime efficiency and scalability of our algorithm. Three IM algorithms are chosen as baselines: the Frequent Interest Matching algorithm (FIM) [15], the Space-Time Interest Matching algorithm (STIM) [2] and the Predictive Interest Matching algorithm using Brute Force approach (PIM-BF).

The rest of this paper is organized as follows. Section 2 briefly describes the existing IM algorithms and discusses their advantages and disadvantages. In section 3, the prediction of the space-time intersection of regions in our algorithm is presented. Section 4 describes the region pruning process of our algorithm in detail. In section 5, the implementation of the PIM algorithm is discussed. Section 6 presents the experimental results of the proposed algorithm and a comparison analysis with the existing matching algorithms. In section 7, issues for discussion are presented. Finally, Section 8 concludes the paper.

\section{Related work}

In this section, we briefly describe the existing IM algorithms. Their advantages and disadvantages are also discussed briefly.

\subsection{Interest matching algorithms}

In recent years, a number of IM algorithms have been proposed for distributed simulation or DVE. These algorithms can be generally classified into four categories: the brute force algorithm, the grid-based algorithm, the hybrid-based algorithm and the sort-based algorithm.

The brute force algorithm checks all update-subscription pairs of regions to find the overlapping region pairs [11]. In an early version of this algorithm, the region is defined as the multidimensional rectangle [16-18]. To improve the filtering precision of the algorithm, researchers proposed many different types of regions and conducted experimental comparisons. Those region types include circular [19-20], circular sector [21], hexagon [22-23], and aura types [24]. However, the increase in complex region type introduces more computational overhead. Whatever region type is used in the algorithm, all update-subscription region pairs should be checked to obtain the overlap information at each timestep. Although it is easy to implement and has high filtering precision, its computational complexity is $O(N M)$, where $N$ and $M$ are the number of update regions and subscription regions, respectively. Therefore, it is not a scalable algorithm and has poor runtime efficiency when $N$ and $M$ are large enough.

To reduce the computational overhead of the brute force algorithm, some researchers proposed the grid-based algorithm [25-26]. The virtual space is partitioned into a grid of cells in this algorithm. Firstly, this algorithm calculates the intersections of the update region and the subscription region with cells. Then, it derives the overlapping information of an update region and a subscription region by checking whether they intersect with the same grid cell [4][11]. Although the grid-based algorithm has lower computational complexity than the brute force algorithm and has high runtime efficiency, its filtering precision is lower than that of the brute force algorithm. The subscription entity will receive irrelevant data from the update entity if their associated regions overlap with the same grid cell but do not actually intersect. Another problem of grid-based filtering is how to define the size of cells. The large size of cell can reduce computational overhead but results in the transfer of more irrelevant data. On the other hand, the 
small cell size has high filtering precision but consumes more computational resources. The selection of the cell size is a complex problem and is affected by many factors, such as the size of AOI, network bandwidth, multicast groups and CPU speed [1][27-28]. Ayani et al. have conducted a study to determine the optimal cell size for the grid-based algorithm [28].

The hybrid algorithm makes use of the advantages of the brute force algorithm and the region-based algorithm to derive the overlap information of the update region and the subscription region. It first uses the grid-based algorithm to map all regions to the grid cells to avoid all update-subscription region pairs overlap tests. Then, the brute force algorithm is employed to obtain the exact overlap information of regions in the same grid cell. The first hybrid algorithm is proposed by Tan et al. [29], and a variation of this algorithm is proposed by Boukerche et al. [30]. Although the hybrid algorithm outperforms the region-based algorithm and has higher filtering precision than that of the grid-based algorithm, it still faces the problem of optimizing cell size similar to the grid-based algorithm. Another problem faced by both the hybrid algorithm and the grid-based algorithm is that additional overhead is introduced by testing the overlap of grid cells and regions. Prabhakar and Tao et al. make efforts to build and maintain an object R-tree for querying the overlap information of regions and grid cells [31-32]. However, some study results show that a simple grid-based index algorithm yields better performance than the R-tree-based solution [33-34].

The sort-based algorithm is designed to improve the matching runtime efficiency. The algorithm sorts the endpoints of each dimension of the region on the coordination axis and then computes the intersections of the update region and the subscription region by checking the overlap of each dimension of the regions. Yu et al. proposed a preliminary version of the sort-based algorithm [35]. Raczy et al. improved the implementation of the algorithm, and experimental results show that their algorithm outperforms the region-based and hybrid algorithms [4]. Ke et al. proposed a dynamic sort-based matching algorithm to improve the runtime performance by avoiding re-sorting the endpoints of each dimension for all regions at each timestep [5]. Wang et al. proposed an improved sort-based IM algorithm to decrease the time cost of their algorithm by using a vector to record the overlaps of regions [36].

There are some IM algorithms that have been researched or implemented for HLA DDM. Petty et al. proved that the DDM specifications are equivalently powerful through a transformation from DDM 1.3 to DDM 1516 configurations and a mapping from DDM 1516 to DDM 1.3 configurations [37]. Van Hook et al. proposed a HLA DDM framework for optimizing data flow between federates and developed a RTI prototype implementation [38]. Morse presented multicast grouping heuristic algorithms for HLA DDM and defined the cost function for evaluating the algorithms. Performance analyses of algorithms are also provided [39].

2.2 The missing event problem

The IM algorithms mentioned in section 2.1 either have efficient runtime performance or high filtering precision. However, they still have two disadvantages: the missing-event problem and the redundant matching problem.

The missing event refers to the overlap event that failed to be detected by the IM algorithms [2]. In case the IM algorithm performs region matching at discrete timesteps and overlaps occur when the regions have been moving continuously between time steps, the algorithm cannot report the event and this leads to an incorrect simulation result. Morse and Steinman argued that the missing-event problem was caused by three factors: infrequent interest matching, rapid entity 
movement and small region size [15]. There are three solutions based on the four category algorithms mentioned in section 2.1 for tackling this problem: the frequent interest matching algorithm, the region expansion algorithm and the space-time algorithm. Those three algorithms use one or more of the four IM algorithms to improve their event-capturing ability.

The frequent interest matching algorithm (called FIM in this paper) is designed to catch the missing events in the time dimension. It uses one of the four IM algorithms and improves the event-capturing ability of the algorithm by reducing the time intervals of matching [15]. Essentially, it is a discrete IM algorithm and cannot capture all events occurring during two timesteps if the regions have been moving continuously between time steps. Furthermore, reducing the timestep of matching will introduce heavy computational overhead, which hampers its runtime performance.

Dissimilar with the frequent matching algorithm, the region expansion (aura expansion) algorithm detects the missing events in the space dimension. It expands the update regions or the subscription regions of entities to catch the missing events [15]. Fujimoto also proposed a similar solution [40]. The drawback of this algorithm is that expanding the region will cause many spurious events. The spurious events may result in the simulation entities receiving a large number of irrelevant messages and the degradation of filtering precision.

The space-time algorithm (called STIM in this paper) is proposed by Elvis et al. [2]. Space-time interest matching tackles the missing event problem in the space-time dimension. This algorithm computes the swept volumes of the update region and subscription region at discrete timesteps. Then, it uses the divide-and-conquer method to approximately compute the actual overlap of two regions to report the missing events. This algorithm does not increase the aura size and thus can preserve the filtering precision of interest management. In addition, it does not increase the frequency of interest matching for all regions and has efficient runtime performance. However, the filtering precision of this algorithm depends on the time threshold parameter. The choice of threshold value is application dependent because it controls the size of the smallest subintervals. It is in fact a trade-off between accuracy and efficiency [2].

In addition to these three algorithms, a predictive interest management algorithm based on the predictive modeling of entity movement was proposed by Morgan et al. [9]. This algorithm determines the frequency of message exchange between objects on the likelihood that these objects will influence each other in the near future. However, Morgan et al. did not provide experiments to evaluate their algorithm.

2.3 The redundant matching problem

Redundant matching is the overlap tests that are not necessary but are performed by the IM algorithms. Redundant matching operations degrade the runtime performance of the algorithm. For example, to capture the missing events, the FIM algorithm must perform a large number of overlap tests. However, some of these overlap tests are unnecessary.

Furthermore, because the existing discrete IM algorithms cannot predict the exact time intervals during which two regions overlap with each other, they always need to perform matching at each timestep. Actually, many matching operations of these algorithms are redundant. This matching cannot report any new overlap information of the regions. For example, let us consider the regions of two entities that move in a 2-dimension virtual space, as shown in Fig. 1. 

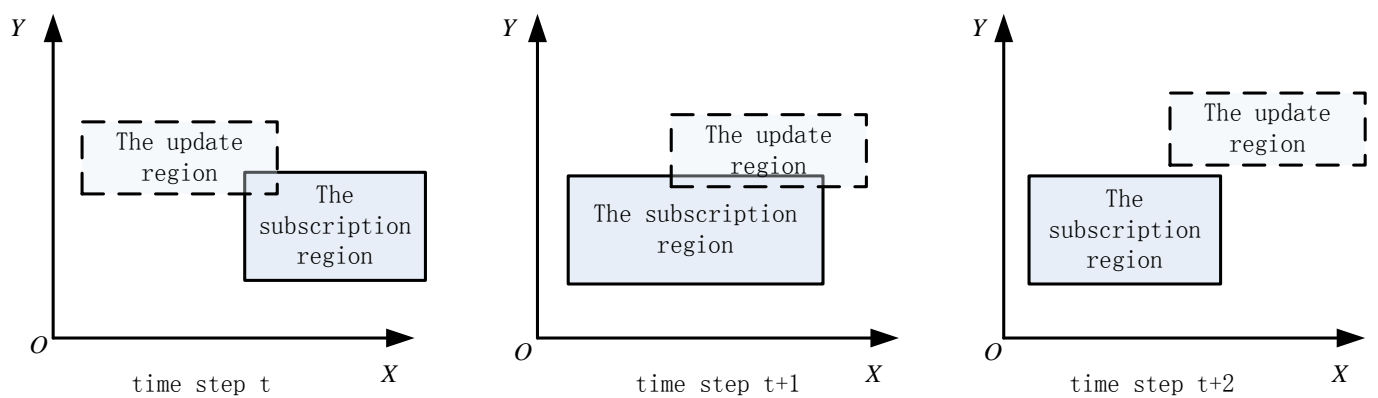

Fig. 1. An example of the redundant matching

At the timestep $t$, the IM algorithm discovers that these two regions overlap with each other. The algorithm performs matching again at timestep $t+1$ but still reports the overlap of the two regions as same as timestep $t$. The algorithm discovers the non-overlap of the two regions until timestep $t+2$. If the IM algorithm can predict that two regions will overlap at timesteps $t$ and $t+1$, it does not need to perform the matching at time step $t+1$. Therefore, the matching at time step $t+1$ is redundant matching.

\section{Predictive model of the space-time intersection for two regions}

\subsection{Model and assumption}

The region in our algorithm is defined as the $K$-dimensional rectangle, which is similar to the definition of the extent of Data Distribution Management (DDM) in HLA [4]. The formal definition of the region in $K$-dimensional space is as follows:

Definition 1: A region $R$ can be defined as $R=\left[l_{1}, u_{1}\right) \times\left[l_{2}, u_{2}\right) \times \cdots \times\left[l_{K}, u_{K}\right)$, where $l_{i}<u_{i}$ for each $i=1,2, \cdots, K, K$ is the number of dimensions, $\mathbf{R}$ is the real number domain ${ }^{2}, l_{i}$ is the lower bound of the region in the ith dimension and $u_{i}$ is the upper bound of the region in the ith dimension. $\left[l_{i}, u_{i}\right)$ is a semi-open interval and is called the range of the region in the dimension $i$.

The update region and the subscription region are denoted as $R^{U}$ and $R^{s}$, respectively. According to the concept of dimension reduction [41] and the sort-based matching algorithm [4], the sufficient and necessary condition for the overlap of two regions in the $N$-dimensional space is that their ranges on each dimension overlap with each other. The overlap of two regions is illustrated in Fig. 2. The update region $R^{U 1}$ and the subscription region $R^{s 1}$ overlap due to their two ranges on each dimension overlapping with each other. For the update region $R^{U 2}$ and the subscription region $R^{s 1}$, they do not overlap with each other because only their ranges in the $1^{s t}$-dimension overlap, but the ranges in the $2^{e d}$-dimension do not overlap. The overlap of two ranges can be formally defined in Lemma 1[4].

Lemma 1: The overlap of the range $\left[l_{i}^{R^{U}}, u_{i}^{R^{U}}\right)$ for an update region $R^{U}$ and the range $\left[l_{i}^{R^{S}}, u_{i}^{R^{S}}\right)$ for a subscription region $R^{S}$ is defined as $\left[l_{i}^{R^{L^{U}}}, u_{i}^{R^{U}}\right) \cap\left[l_{i}^{R^{S}}, u_{i}^{R^{S}}\right) \neq \varnothing$. Then

$$
\left[l_{i}^{R^{U^{U}}}, u_{i}^{R^{U}}\right) \cap\left[l_{i}^{R^{s}}, u_{i}^{R^{s}}\right) \neq \varnothing \Leftrightarrow l_{i}^{R^{U}}<u_{i}^{R^{s}} \wedge l_{i}^{R^{s}}<u_{i}^{R^{U}} .
$$

As mentioned above, the overlap of two regions can be judged according to Lemma 2.

Lemma 2: Two regions $R^{U}$ and $R^{S}$ overlap with each other and are defined as $R^{U} \cap R^{S} \neq \varnothing$. Then

$$
\left.R^{U} \cap R^{S} \neq \varnothing \Leftrightarrow\left[l_{1}^{R^{U}}, u_{1}^{R^{U}}\right) \cap\left[l_{1}^{R^{S}}, u_{1}^{R^{S}}\right) \neq \varnothing \wedge\left[l_{2}^{R^{U}}, u_{2}^{R^{U^{U}}}\right) \cap\left[l_{2}^{R^{S}}, u_{2}^{R^{S}}\right) \neq \varnothing \wedge \ldots \wedge l_{K}^{R^{U}}, u_{K}^{R^{U}}\right) \cap\left[l_{K}^{R^{S}}, u_{K}^{R^{S}}\right) \neq \varnothing .
$$

2 All real numbers in this paper are expressed by 64-bit floating point numbers on a digital computer.

The precision of numbers is limited by computer. 


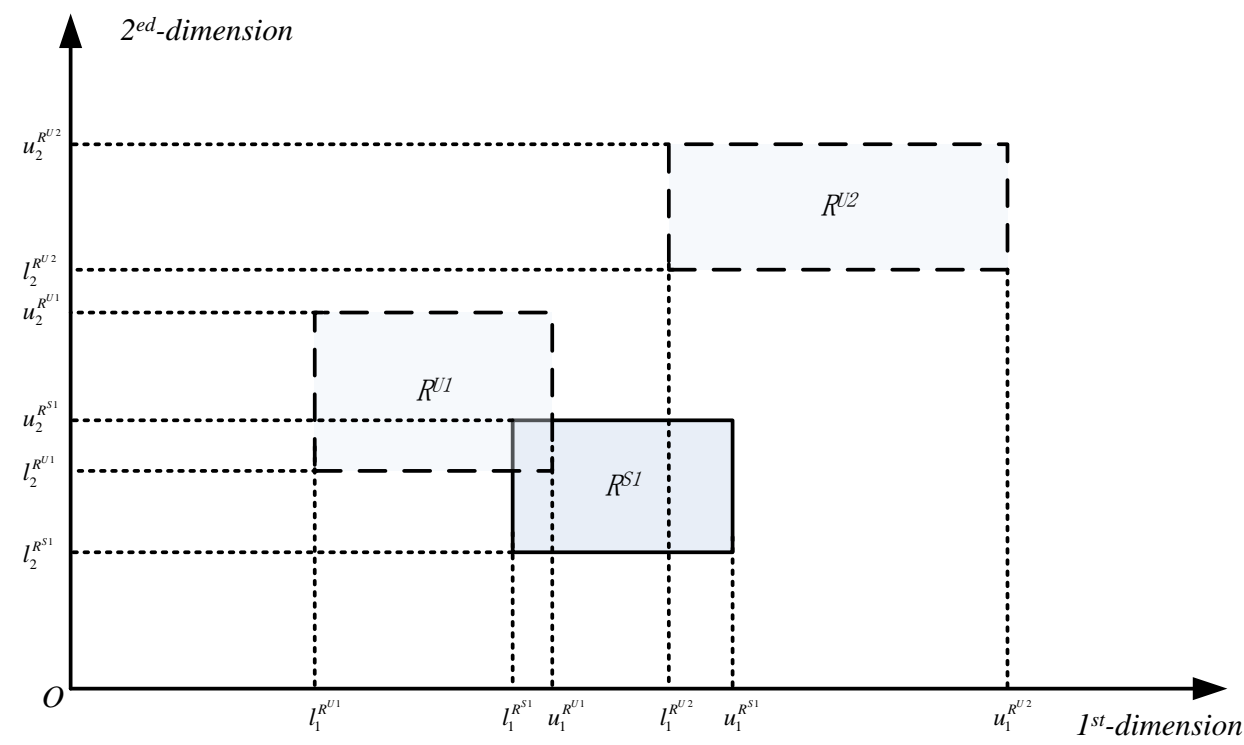

Fig. 2. The overlap of two regions

In distributed simulation or DVE, movements of the virtual entity can be very complex. Modeling the movements of an entity precisely is time consuming and unnecessary. A common approach to model the interpolation of entity movement is to use polynomial functions [2][42]. These techniques have been widely utilized in military training simulations [44-46] and networked games [42][47-53]. We assume that there is a polynomial function $\vec{Y}(t)$ that describes the movement of the virtual entity during the time interval $\left[t_{a}, t_{b}\right]$. We call $\left[t_{a}, t_{b}\right]$ the predictive time interval. For example, $\vec{Y}(t)$ can be expressed as equation (1) in two-dimension Cartesian space

$$
\vec{Y}(t)=\vec{Y}_{t}\left(d+x_{1} t \hat{i}(+)_{2} t \hat{j}=(x) \hat{i}+y(t) \hat{\jmath}\right.
$$

where $\hat{i}$ and $\hat{j}$ are the unit vectors and $x$ and $y$ are the coordinates of the virtual entity, which vary with time $t$ according to the polynomial movement functions $x_{1}(t)$ and $x_{2}(t)$, respectively.

We assume that the relative position of the region with the virtual entity is fixed during the predictive time interval. If the relative position of region with the virtual entity can change during a predictive time interval, the interval should be divided into sub-intervals until the relative position is fixed. Therefore, the movement of the lower bound and the upper bound of ranges of the region associated with a virtual entity can also be described by the polynomial motion functions of the virtual entity, respectively.

$$
\begin{array}{r}
l_{i}(t)=l_{i}\left(t_{a}\right)+x_{i}(t) \\
u_{i}(t)=u_{i}\left(t_{a}\right)+x_{i}(t)
\end{array}
$$

If we can exactly compute the time intervals for the overlap of ranges in each dimension, we can know when two regions overlap during $[a, b]$ according to Lemma 1 and Lemma 2. This process needs to solve the polynomial inequation. If the degree of the polynomial movement function $\operatorname{deg}\left(x_{i}(t)\right)$, is greater than 2, solving higher-order polynomial inequations can be very expensive and the numerical solution results are inaccurate. Therefore, we assume that the polynomial movement function $x_{i}(t)$ of the virtual entity is a quadratic polynomial and can be expressed as (4).

$$
x_{i}(t)=v_{i}\left(t_{a}\right) t+0.5 a_{i} t^{2}
$$

where $v_{i}\left(t_{a}\right) \in \mathbf{R}$ is the velocity in dimension $i$ of the virtual entity and $a_{i} \in \mathbf{R}$ is the acceleration of the 
virtual entity in the dimension $i$. The acceleration $a_{i}$ is a constant during $\left[t_{a}, t_{b}\right]$.

\subsection{Overlap prediction of two ranges}

As mentioned in section 3.1, to compute the time interval(s) for the overlap of two regions during $\left[t_{a}, t_{b}\right]$, it is necessary to obtain the time interval(s) for the overlap of ranges in each dimension. For the update region $R^{U}$ and the subscription region $R^{s}$, according to equations (2), (3), and (4), the polynomial functions describing the motions of their lower bounds and upper bounds in dimension $i$ can be described as follows.

$$
\begin{aligned}
& l_{i}^{R^{n}}(t)=l_{i}^{R^{U}}\left(t_{a}\right)+v_{i}^{R^{U}}\left(t_{a}\right) t+0.5 a_{i}^{R^{U}} t^{2} \\
& u_{i}^{R^{U}}(t)=u_{i}^{R^{U}}\left(t_{a}\right)+v_{i}^{R^{U}}\left(t_{a}\right) t+0.5 a_{i}^{R^{U}} t^{2} \\
& l_{i}^{R^{s}}(t)=l_{i}^{R^{s}}\left(t_{a}\right)+v_{i}^{R^{s}}\left(t_{a}\right) t+0.5 a_{i}^{R^{s}} t^{2} \\
& u_{i}^{R^{s}}(t)=u_{i}^{R^{s}}\left(t_{a}\right)+v_{i}^{R^{s}}\left(t_{a}\right) t+0.5 a_{i}^{R^{s}} t^{2}
\end{aligned}
$$

Assuming the ranges overlap during the time interval $\left[t_{1}, t_{2}\right] \subseteq\left[t_{a}, t_{b}\right]$, according to Lemma 1, we know that for $\forall t \in\left[t_{1}, t_{2}\right]$, the inequations are as follows.

$$
\left\{\begin{array}{l}
u_{i}^{R^{U}}(t)-l_{i}^{R^{S}}(t)=u_{i}^{R^{U}}\left(t_{a}\right)+v_{i}^{R^{U}}\left(t_{a}\right) t+0.5 a_{i}^{R^{U}} t^{2}-\left(l_{i}^{R^{S}}\left(t_{a}\right)+v_{i}^{R^{S}}\left(t_{a}\right) t+0.5 a_{i}^{R^{S}} t^{2}\right)>0 \\
u_{i}^{R^{S}}(t)-l_{i}^{R^{U}}(t)=u_{i}^{R^{S}}\left(t_{a}\right)+v_{i}^{R^{S}}\left(t_{a}\right) t+0.5 a_{i}^{R^{S}} t^{2}-\left(l_{i}^{l^{U}}\left(t_{a}\right)+v_{i}^{R^{U}}\left(t_{a}\right) t+0.5 a_{i}^{R^{U}} t^{2}\right)>0
\end{array}\right.
$$

Let $q(t)=u_{i}^{R^{U}}(t)-l_{i}^{R^{S}}(t), p(t)=u_{i}^{R^{S}}(t)-l_{i}^{R^{U}}(t), A=0.5 a_{i}^{R^{R^{U}}}-0.5 a_{i}^{R^{S}}, B=v_{i}^{R^{R^{U}}}\left(t_{a}\right)-v_{i}^{R^{s}}\left(t_{a}\right), C=u_{i}^{R^{U}}\left(t_{a}\right)-l_{i}^{R^{S}}\left(t_{a}\right)$, $D=u_{i}^{R^{S}}\left(t_{a}\right)-l_{i}^{R^{U}}\left(t_{a}\right)$. Then, inequation (9) can be rewritten as follows.

$$
\left\{\begin{array}{c}
q(t)=A t^{2}+B t+C>0 \\
p(t)=-A t^{2}-B t+D>0
\end{array}\right.
$$

To compute $\left[t_{1}, t_{2}\right]$, which satisfies inequation (10), we first find the roots of equations (11) and (12) in the real number domain.

$$
\begin{aligned}
& q(t)=A t^{2}+B t+C=0 \\
& p(t)=-A t^{2}-B t+D=0
\end{aligned}
$$

Once we know the roots of equations (11) and (12), we can obtain the exact time interval(s), which satisfies inequation (9). To solve equations (11) and (12), it is necessary to consider three different cases: $A \neq 0, A=0$ and $B \neq 0, A=0$ and $B=0$.

Case 1: $A \neq 0$

In the case of $A \neq 0$, equation (11) has no root or two roots (the same two roots are considered as two roots in this paper). Therefore, there are at most four roots for $q(t)=0$ and $p(t)=0$. We assume that the four roots are denoted as $r_{1}, r_{2}, r_{3}, r_{4}$ and satisfy $r_{1} \leq r_{2} \leq r_{3} \leq r_{4}$. According to equations (11) and (12), we know that $p(t)$ is not independent with $q(t)$ and that they have the relation as follows.

$$
\begin{aligned}
& p(t)=-q(t)+C+D=-q(t)+u_{i}^{R^{U}}\left(t_{a}\right)-l_{i}^{R^{S}}\left(t_{a}\right)+u_{i}^{R^{S}}\left(t_{a}\right)-l_{i}^{R^{U}}\left(t_{a}\right)=-q(t)+u_{i}^{R^{U}}\left(t_{a}\right)-l_{i}^{R^{U}}\left(t_{a}\right)+u_{i}^{R^{S}}\left(t_{a}\right)-l_{i}^{R^{S}}\left(t_{a}\right) \\
& =-q(t)+\text { range } e_{i}^{R^{U}}+\text { range }_{i}^{R^{s}}
\end{aligned}
$$

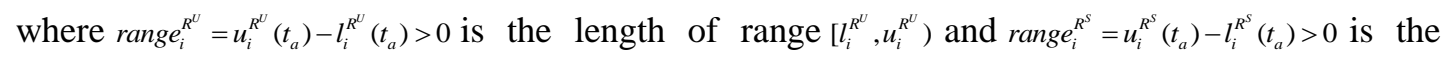
length of range $\left[l_{i}^{R^{s}}, u_{i}^{R^{s}}\right)$. Therefore, the roots of equation (12) are not independent with the roots of equation (11).

Fig. 3 illustrates the relation of the roots of equations (11) and (12). The polynomial $q(t)$ and $p(t)$ can be exchanged with each other. When there are four, two roots (both two roots for equations (11) and (12)), as shown in Fig. 3 (a), the time intervals $\left[r_{1}, r_{2}\right]$ and $\left[r_{3}, r_{4}\right]$ satisfy inequation (10), i.e., the range ${ }_{i}^{R^{U}}$ overlaps with the range $_{i}^{R^{s}}$. These two ranges begin to overlap with each other at $r_{1}$ and are apart at $r_{2}$. We say that $r_{1}$ is the point of time of intersection (PTI), and $r_{2}$ is the point of time of separation (PTS). Therefore, the root $r_{3}$ is a PTI and the root $r_{4}$ is a PTS. 


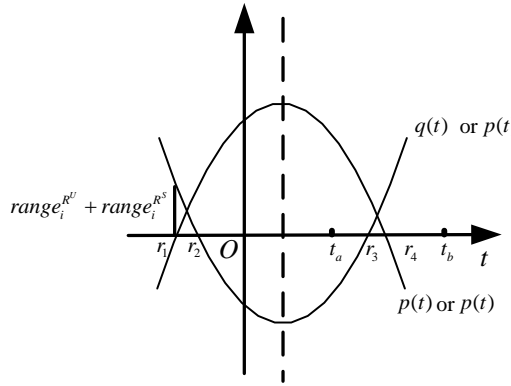

(a). Four roots

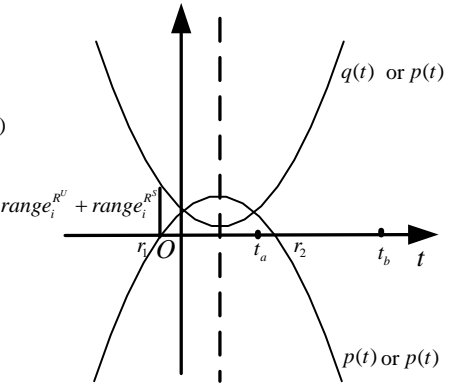

(b). Two roots

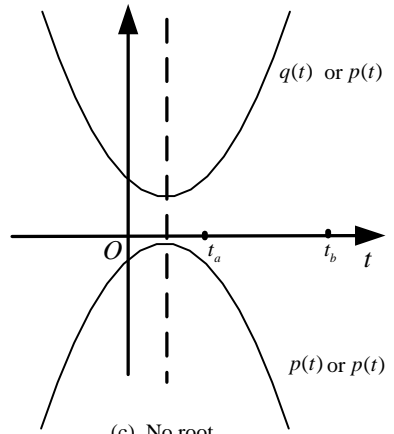

(c). No root

Fig. 3. Time intervals for overlap of two ranges for $A \neq 0$

In case only $q(t)=0$ or $p(t)=0$ has two roots $r_{1}$ and $r_{2}$, as illustrated in Fig. 3 (b), range $e_{i}^{R^{u}}$ overlaps with range $_{i}^{R^{S}}$ during the time interval $\left[r_{1}, r_{2}\right]$. The root $r_{1}$ is a PTI, and the root $r_{2}$ is a PTS. In case there is no root for $q(t)=0$ and $p(t)=0$, range $e_{i}^{R^{U}}$ and range $_{i}^{R^{S}}$ never overlap with each other, which is shown in Fig. 3(c). The overlap time interval is empty.

The relationship for a different number of roots and the overlap time interval(s) is shown in Table 1. In the case of no root, the overlap time interval is empty. If there are two roots for equations (11) and (12), the overlap time interval is $\left[r_{1}, r_{2}\right]$. For the case of four roots, there are two time intervals $\left[r_{1}, r_{2}\right]$ and $\left[r_{3}, r_{4}\right]$. Then, we can obtain the overlap time intervals that belong to $\left[t_{a}, t_{b}\right]$ by computing the intersection of $\left[t_{a}, t_{b}\right]$ and the overlap time interval(s) defined by the roots. For example, in Fig. 3, the overlap time intervals that belong to $\left[t_{a}, t_{b}\right]$ are $\left[t_{a}, r_{2}\right]$ and $\left[r_{3}, r_{4}\right]$ for the case of two roots and the case of four roots, respectively.

Table 1. The relation of roots and overlap time interval(s) for $A \neq 0$

\begin{tabular}{|c|c|c|c|c|c|l|}
\hline Roots & $r_{1}$ & $r_{2}$ & $r_{3}$ & $r_{4}$ & $\begin{array}{c}\text { Overlap time } \\
\text { interval(s) }\end{array}$ & $\begin{array}{l}\text { Intersection of overlap time } \\
\text { interval with }[a, b] \text { in Fig. 3 }\end{array}$ \\
\hline No root & & & & & empty & empty \\
\hline Two roots & PTI & PTS & & & {$\left[r_{1}, r_{2}\right]$} & {$\left[t_{a}, r_{2}\right]$} \\
\hline Four roots & PTI & PTS & PTI & PTS & {$\left[r_{1}, r_{2}\right],\left[r_{3}, r_{4}\right]$} & {$\left[r_{3}, r_{4}\right]$} \\
\hline
\end{tabular}

Case 2: $A=0, B \neq 0$

In the case of $A=0$ and $B \neq 0$, equations (11) and (12) degrade to the linear equations but still satisfy equation (13). The relation of $q(t)$ and $p(t)$ is shown in Fig. 4. Equations (11) and (12) have one root, $r_{1}$ and $r_{2}$, respectively. We assume that $r_{1}$ and $r_{2}$ satisfy $r_{1} \leq r_{2}$. Therefore, the overlap time interval is $\left[r_{1}, r_{2}\right]$. Similar to the case of $A \neq 0$, the relation of roots and overlap time interval(s) is shown in Table. 2. Then, the overlap time interval belonging to $\left[t_{a}, t_{b}\right]$ is $\left[t_{a}, r_{2}\right]$, as shown in Fig. 4.

Table 2 The relation of roots and overlap time interval(s) for $A \neq 0$

\begin{tabular}{|c|c|c|c|c|}
\hline Roots & $r_{1}$ & $r_{2}$ & Overlap time interval & $\begin{array}{c}\text { Intersection of overlap time } \\
\text { interval with }[a, b] \text { in Fig. } 4\end{array}$ \\
\hline Two roots & PTI & PTS & {$\left[r_{1}, r_{2}\right]$} & {$\left[t_{a}, r_{2}\right]$} \\
\hline
\end{tabular}

Case 3: $A=0, B=0$

In the case of $A=0$ and $B=0$, the virtual entities remain stationary during $\left[t_{a}, t_{b}\right]$. Therefore, both range $_{i}^{R^{U}}$ and range $_{i}^{R^{s}}$ do not move during the predictive time interval $\left[t_{a}, t_{b}\right]$. If these two ranges 
overlap with each other at the point of time $a$, they overlap with each other during the whole time interval $\left[t_{a}, t_{b}\right]$. On the contrary, they will never overlap with each other at any point of time in $\left[t_{a}, t_{b}\right]$ if they are away from each other at the point of time $a$.

According to cases 1,2 and 3, the overlap time interval(s) in the real number domain for two ranges can be exactly obtained. The intersection of these time interval(s) with the predictive time interval $\left[t_{a}, t_{b}\right]$ is the actual overlap time interval(s) of two ranges. We denote the set of overlap time intervals contained in $\left[t_{a}, t_{b}\right]$ for two ranges in the ith dimension as IntervalSet $t_{i}$.

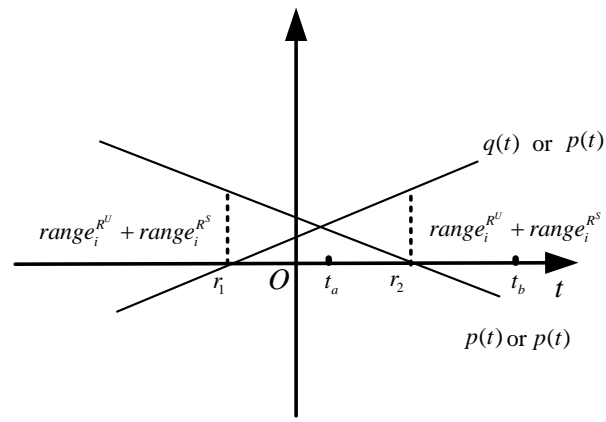

Fig. 4. Time intervals for overlap of two ranges for $A=0, B \neq 0$

\subsection{Overlap prediction of two regions}

The computation of the overlap time interval for two ranges on one dimension is solved as mentioned in section 3.2. It is easy to prove that the set of overlap time intervals of two regions (denoted as ROTI) is the intersection of all IntervalSet $t_{i}$ for each dimension. The ROTI can be formally denoted as follows.

$$
R O T I=\bigcap_{i=1}^{n} \text { IntervalSet }_{i}
$$

The intersection of IntervalSet $t_{i}$ and IntervalSet ${ }_{j}$ (denoted as IntervalSet ${ }_{i} \cap$ IntervalSet $_{j}$ ) is defined as the set of intersection of two arbitrary time intervals. One of these two time intervals is selected from IntervalSet $_{i}$, and the other belongs to IntervalSet ${ }_{j}$. For example, in Fig. 5, the predictive time interval is $[a, b]=[1.0,21.2]$. The sets of overlap time intervals for ranges in the three-dimension space are IntervalSet $t_{1}=\{[2.0,7.0],[12.0,16.5]\}, \quad$ IntervalSet $_{2}=\{[5.8,13.2]\} \quad$ and IntervalSet $_{3}=\{[2.0,6.7],[9.6 .17 .9]\}$, respectively. The intersection of overlap time interval(s) on ranges on the $1^{s t}$ dimension and $2^{e d}$ dimension is $\{[5.8,7.0],[12.0,13.2]\}$. Then, the intersection of $\{[5.8,7.0],[12.0,13.2]\}$ and Intervalset ${ }_{3}$ is $[5.8,6.7]$. Therefore, these two regions overlap with each other during [5.8,6.7] , namely, $R O T I=\{[5.8,6.7]\}$.

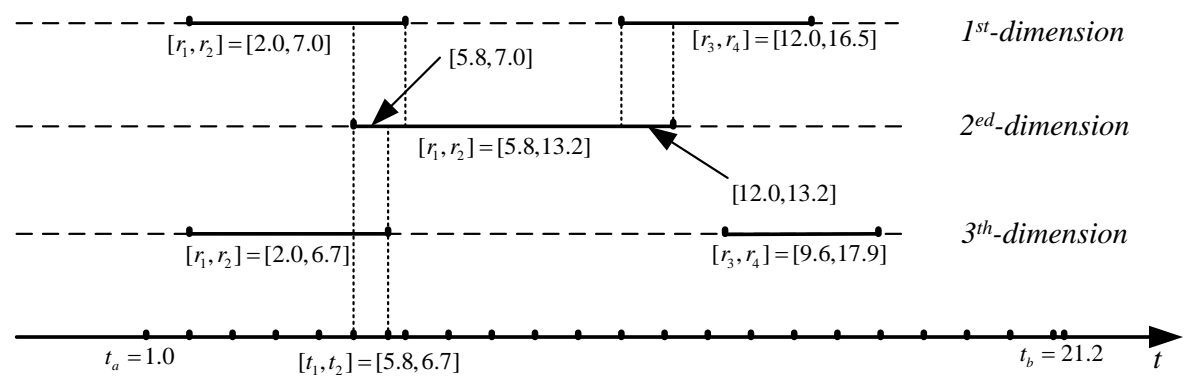

Fig. 5. Intersection of overlap time intervals for three-dimension space 
For the sake of simplification, we assume that the predictive time interval $\left[t_{a}^{R^{U}}, t_{b}^{R^{t^{U}}}\right]$ of update region and the predictive time interval $\left[t_{a}^{R^{S}}, t_{b}^{R^{S}}\right]$ of subscription region are the same in above. This is not true under most situations. Therefore, the difference between $\left[t_{a}^{R^{U}}, t_{b}^{R^{R^{U}}}\right]$ and $\left[t_{a}^{R^{s}}, t_{b}^{R^{s}}\right]$ is allowed in the matching computing of two regions. The overlap prediction of two regions is called ROTIComputing and is illustrated in Algorithm 1. If $\left[t_{a}^{R^{U}}, t_{b}^{R^{D^{U}}}\right]$ and $\left[t_{a}^{R^{s}}, t_{b}^{R^{s}}\right]$ are different, the lower bound of the predictive time interval of two regions is the maximum of $t_{a}^{R^{U}}$ and $t_{a}^{R^{s}}$. The upper bound of the predictive time interval of two regions is the minimum of $t_{b}^{R^{n}}$ and $t_{b}^{R^{s}}$. The process of calculating the predictive time interval is shown as Lines 2-3 in Algorithm 1. The process of ROTI involves determining the intersection of intervals across all $K$ dimensions, which is shown as Lines 25-26. For each interval in the first interval set IntervalSet $t_{1}$, the intersection(s) of the interval and each interval in ROTI is calculated. Then, the results are used to substitute the interval(s) in ROTI . Finally, repeat those two steps for the remaining interval sets. Fig.5 is an example describing this process.

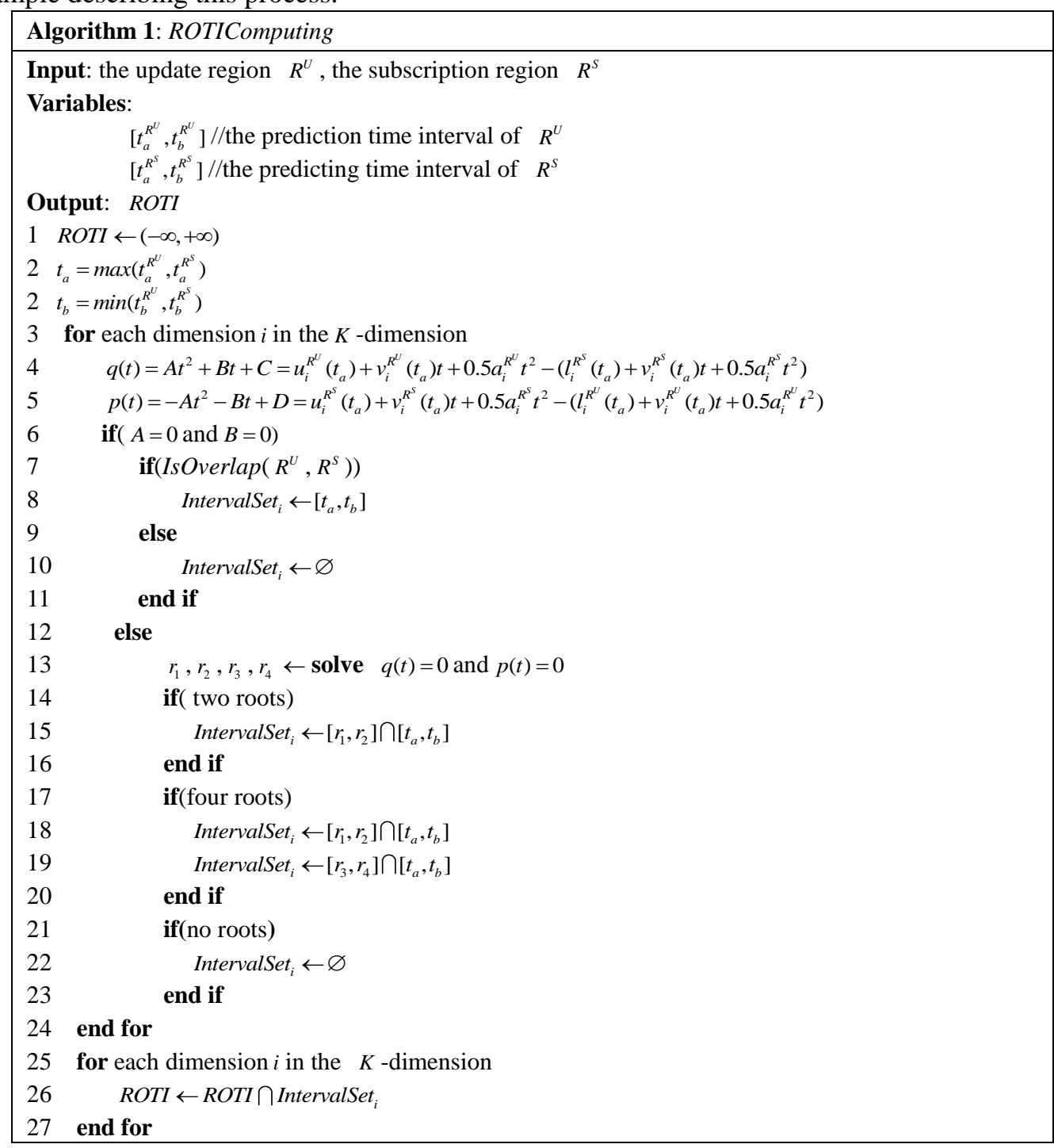

\section{Potential overlap regions and region pruning}

The ROTIComputing algorithm can exactly capture all overlap events occurring during the predictive time interval for two regions. Then, the brute force algorithm can be used to select every update-subscription region pairs to implement interest matching for all regions. However, it 
is obvious that the brute force algorithm is not the optimum algorithm because some update regions may never overlap with some subscription regions during the predictive time interval. In this section, we use Maximum Area of Influence (MAI) based on the movement prediction of a region to acquire the potential overlap regions of the region. With this method, our algorithm can cull out the region pairs that are unlikely to overlap with each other during the predictive time interval. The MAI method is similar to the swept volume method, which is proposed by Liu et al. [2].

\subsection{Maximum area of influence}

The concept of MAI is illustrated in Fig. 6. In two-dimension virtual space, there are two regions: the update region $R^{U}$ and the subscription region $R^{s}$. Their movement tracks in the virtual space during the predictive time interval $\left[t_{a}, t_{b}\right]$ is shown in Fig. 6 (a). From time $t_{a}$ to time $t_{b}$, each region sweeps an area in the virtual space. The swept area is a two-dimension rectangle. This area is the $M A I$ of the region. The $M A I$ of $R^{U}$ is the blue rectangle, denoted as $M I A^{R^{V}}$, and is shown as Fig. 6 (b). The $M I A^{R^{S}}$ is the MAI of $R^{S}$ and is shown as the red rectangle in Fig. 6 (b). In fact, the MAI of a region can also be defined as a region. The formal definition of the $M A I$ of a region during the predictive time interval $\left[t_{a}, t_{b}\right]$ is as follows.

$$
M I A=\left\{\left[\operatorname{minl}_{i}, \operatorname{maxu}_{i}\right] \mid \operatorname{minl}_{i}=\min _{\left.t \in t_{a}, t_{b}\right]}\left(l_{i}(t)\right), \operatorname{maxu}_{i}=\max _{\left.t \in t_{a}, t_{b}\right]}\left(u_{i}(t)\right)\right\}
$$

If the maximum area of influence $M I A^{R^{s}}$ of a subscription region $R^{s}$ and the maximum influence area $M I A^{R^{U}}$ of a publish region $R^{U}$ do not overlap with each other, the region $R^{S}$ and the region $R^{U}$ will not overlap with each other during the time interval $\left[t_{a}, t_{b}\right]$.

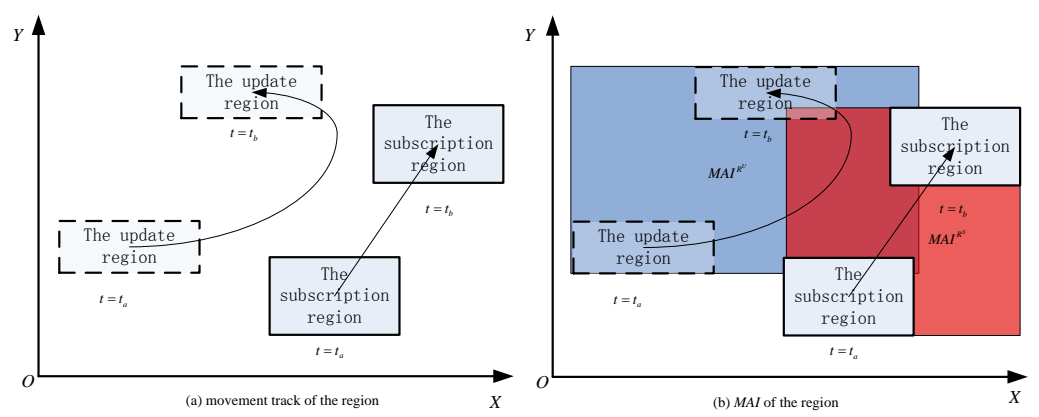

Fig. 6. The maximum influence area of the region

\subsection{Region pruning}

Let us consider that there are one update region and $M$ subscription regions in virtual space ( $M$ may be a very large integer). The entity associated with the update region updates movement polynomial and requires to perform overlap tests during predictive time interval $\left[t_{a}, t_{b}\right]$. If we directly use ROTIComputing algorithm (Algorithm 1) to test overlaps for every region pair, we should perform matching operations for $M$ region pairs. However, the update region may only overlap with a very small subset of $M$ subscription regions. Although ROTIComputing algorithm is faster, it is a waste of CPU cycles to perform matching operations for region pairs that are unlikely to overlap with each other. Therefore, the region pairs that will certainly not overlap during the predictive time interval $\left[t_{a}, t_{b}\right]$ should be removed before performing accurate overlap tests. Region pruning method is employed to eliminate region pairs that are unlikely to overlap with each other. The region pruning method uses sort-based IM algorithm to test the overlap of MAIs of region pairs. If the MAIs are not overlapped with each other, the region pair can not 
overlap during the time interval $\left[t_{a}, t_{b}\right]$. Through region pruning, region pairs that potentially overlap are known. Then, accurate overlap calculation should be performed to find out which region pair is actually overlapped. Although sort-based IM algorithm is faster than ROTIComputing algorithm, sort-based IM algorithm can not be applied to perform accurate overlap test for region pairs during the predictive time interval $\left[t_{a}, t_{b}\right]$ due to the missing event problem. Therefore, ROTIComputing algorithm is used to perform accurate overlap tests for region pairs.

Let $U_{N}$ and $S_{M}$ be the set of update regions and subscription regions, respectively. The sets $U_{N}$ and $S_{M}$ are represented as $U_{N}=\left\{R_{j}^{U} \mid 1 \leq j \leq N\right\}$ and $S_{M}=\left\{R_{k}^{S} \mid 1 \leq k \leq M\right\} . N$ and $M$ are the number of update regions and subscription regions, respectively. The process of region pruning consists of two phases. In the first phase, we compute the maximum area of influence for each region in $U_{N}$ and $S_{M}$ according to the predictive time interval $\left[t_{a}, t_{b}\right]$.

In the second phase, we evaluate the overlap state of maximum influence areas for each update-subscribe region pair. As mentioned in section 4.1, the maximum area of influence of a region is also a region in $N$-dimensional space. We therefore use the sort-based matching algorithm [4][34] to test the overlap of the maximum area of influence. The test results are used to conduct the matching computation for $R_{j}^{U}$ and $R_{k}^{s}$. If the maximum areas of influence of $R_{j}^{U}$ and $R_{k}^{s}$ do not overlap, it is not necessary to test whether these two regions overlap. Otherwise, $R_{j}^{U}$ and $R_{k}^{s}$ potentially overlap with each other. Additionally, more accurate overlap results can be obtained via the ROTIComputing algorithm.

Through region pruning, the region pairs that will certainly not overlap during the predictive time interval can be removed before performing accurate overlap tests. In case there are a large number of regions in virtual space, region pruning can avoid a large amount of unnecessary matching computation.

\subsection{The PIM algorithm}

According to the region pruning and overlap prediction of two regions mentioned in the previous section, we present our PIM algorithm for the set of update regions $U_{N}$ and that of subscription region $S_{M}$. The pseudo-code for the PIM algorithm is illustrated in Algorithm 2. Once one entity updates its movement polynomial or regions associated with it, the regions are updated. Then, the updated region is inserted into the replanRegionSet (a set data structure for storing the regions that need to re-compute the MAI, see lines: 1-10). Then, the MAI of each region within replanRegionSet is computed afresh according to the new predictive time interval and the new polynomial $\vec{Y}^{(t)}$ (see lines: 11-13).

To obtain the region pairs that potentially overlap with each other, we test the overlap state of the MAIs of regions in each region pair (see line: 14). If the MAI of an update region and that of a subscription region overlap with each other, the corresponding region pair would be inserted into the pairSet (see lines: 15-17).

\begin{tabular}{|c|c|}
\hline \multicolumn{2}{|c|}{ Algorithm 2: PIM algorithm } \\
\hline \multirow{2}{*}{\multicolumn{2}{|c|}{$\begin{array}{l}\text { Input: } U_{N} / / \text { the set of update regions } \\
\qquad S_{M} / / \text { the set of subscription regions } \\
\text { Variables: }\end{array}$}} \\
\hline & \\
\hline & pairSet $\leftarrow$ empty//a set of region pairs that are potentially overlapped \\
\hline & replanRegionSet // a set of regions that need to re-compute the $M A I$ \\
\hline Output: & $\begin{array}{l}\text { t: ROTISet //a result set storing pairs of regions that overlap with each other and their time } \\
\text { intervals of overlap }\end{array}$ \\
\hline
\end{tabular}




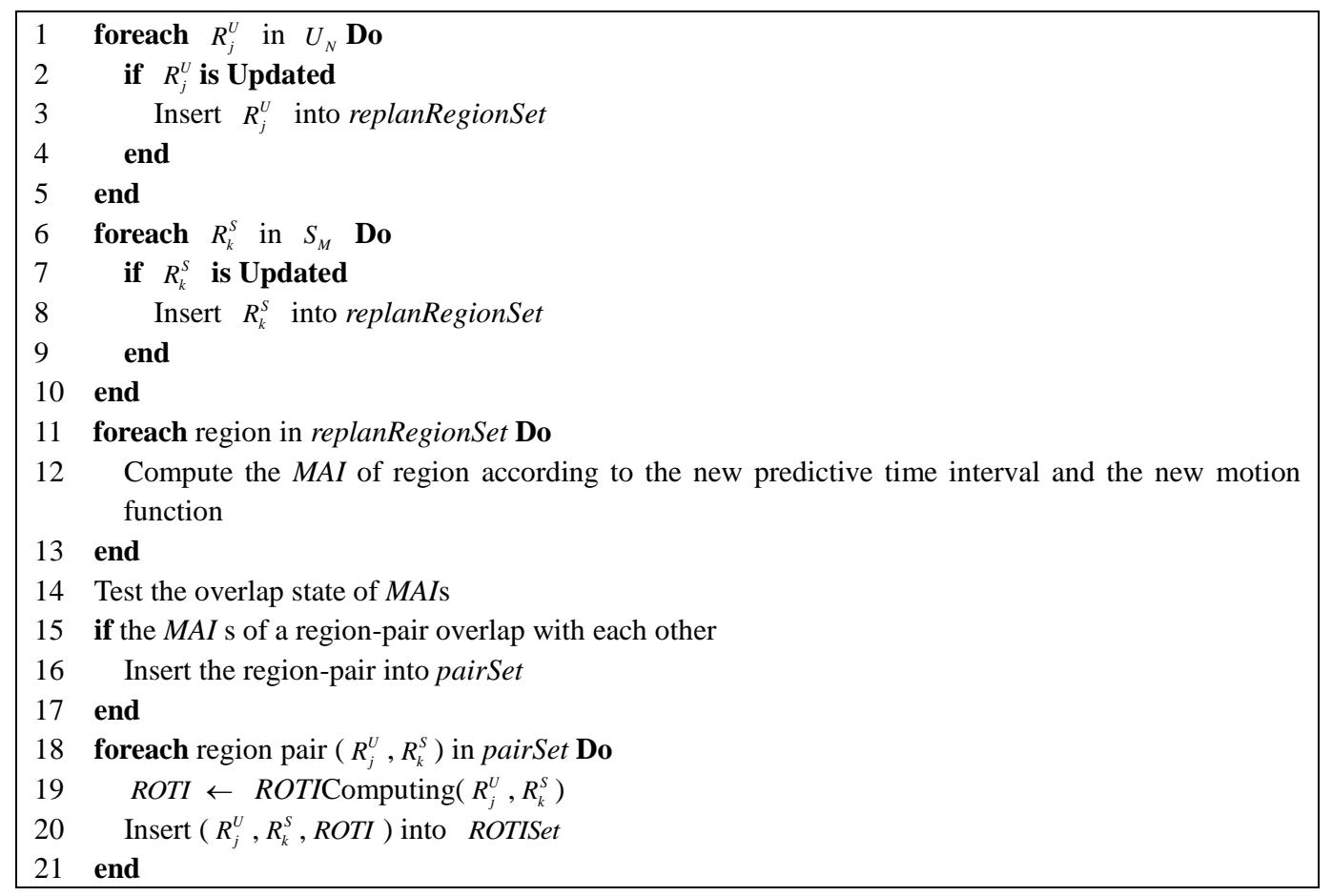

The PIM algorithm is only performed under the circumstance when one entity updates its movement polynomial or regions associated with it. Therefore, matching operations can happen at any point of logical time, and need not to be performed in uniform steps. The result with the PIM algorithm is excessive iterations of the matching operations. Moreover, the PIM algorithm can only calculates the overlaps changed through the region pruning technique. Through region pruning, the region pairs that will certainly not overlap during the predictive time interval can be removed before performing accurate overlap tests. So, The PIM algorithm operates in an incremental way.

\section{Implementation}

Although we have presented the principle of the PIM algorithm and shown its performance, the implementation of the PIM algorithm in distributed simulations or DEVs is still an open issue. To detect overlaps of regions, the PIM algorithm needs to collect all predictive time intervals, update regions, subscription regions and movement polynomials of entities distributed on different computing nodes. However, this requirement conflicts with the inherent distributed characteristic of distributed simulations. Considering the implementation of the PIM algorithm in distributed simulations or DEVs, there are two potential patterns: the distributed pattern and the centralized pattern.

The distributed pattern is suitable for distributed simulations, such as HLA, in which entities are distributed on different computational nodes. As mentioned by Morse, there are three opportunities for date filtering in a simulation system: at sources, on the network via routing, and at receivers. There are different cost tradeoffs involved in each of these [39]. A sources filtering implementation of the PIM algorithm in the HLA is shown in Fig. 7. Because the PIM algorithm relies on movement polynomials of entities to calculate region overlaps and the HLA DDM service does not provide interfaces to process those movement polynomials, a local PIM manager is designed in each federate. A local PIM manager relies on declaration management service and object management service in Local RTI Component (LRC) to transfer data of regions. In a 
federate, LRC communicates with the local PIM manager via application programming interfaces.

The process of data filtering using the PIM algorithm is shown in Fig. 7. First, federate B uses declaration management of its LRC to notify federate A its subscription requirement. Once the LRC of federate A obtains the subscription requirement, the LRC transfers the information of the object class and the computational node hosting federate B to the local PIM manager of federate A. The local PIM manager sets up a virtual link between the source PIM manager (PIM manager in federate A) and the sink PIM manager (the PIM manager of federate B) based on physical connections. Then, federate $\mathrm{B}$ can update subscription regions and send the data of subscription regions to the PIM manager of federate A via the virtual link. Federate A updates data of update regions to its local PIM manager. Once the data of regions is obtained by the source PIM manager, the PIM manager can calculate overlaps through the PIM algorithm. Finally, the source PIM manager can determine whether to send attributes of the entity to sink PIM manager based on the matching results. In case the sink PIM manager receives the attribute update, it sends attributes to federate B.

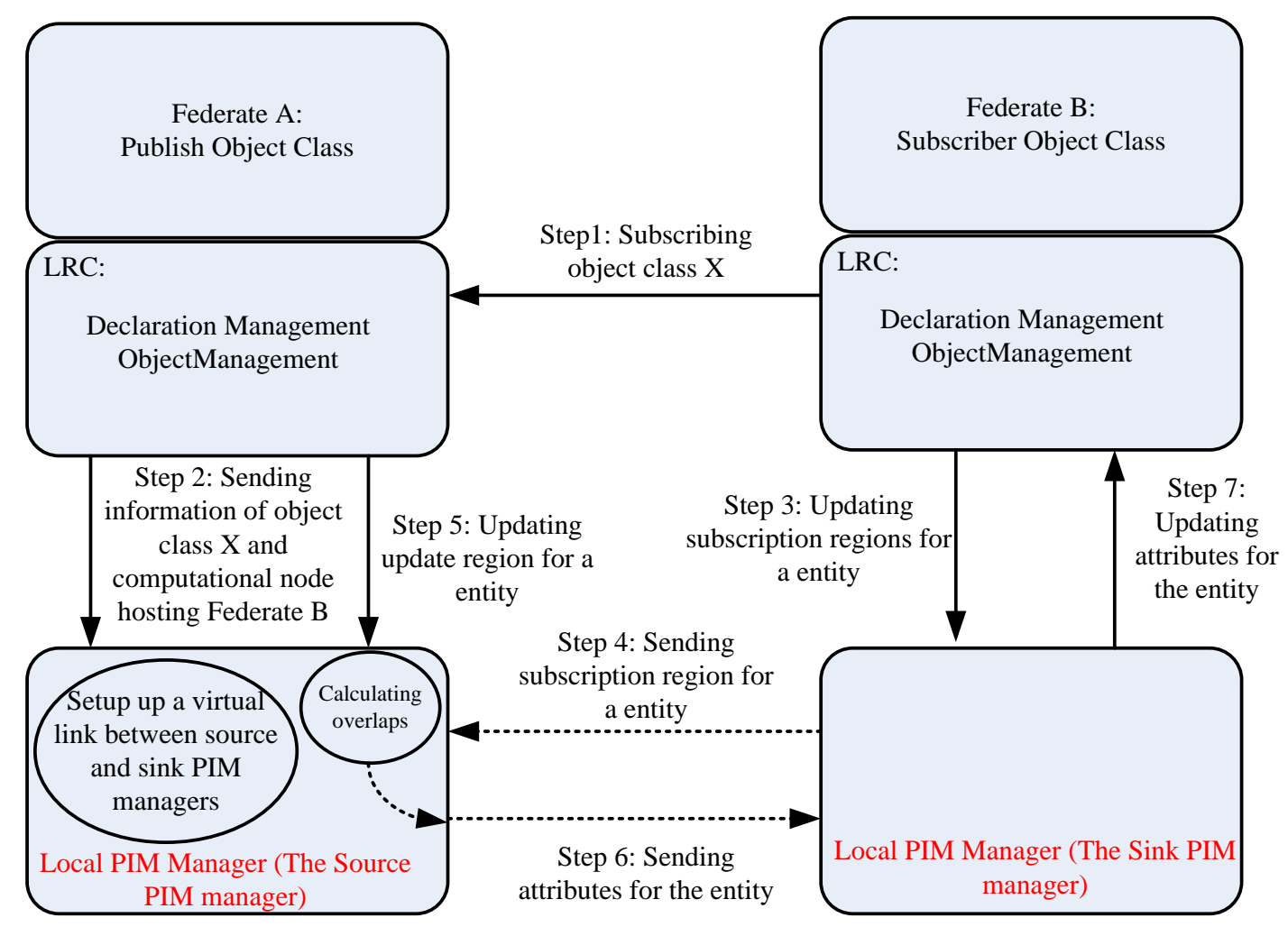

Fig. 7 An implementation of PIM algorithm for HLA

The source filtering implementation of PIM algorithm requires additional protocols to inform update federates about the subscription regions of other federates. The protocol for exchanging messages among local PIM managers of the Federates consists of three parts: initialization, matching, and update. In the initialization part, multicast groups are created by the PIM managers for sending the data of the subscription regions. The point-to-point communication channels between the source PIM managers and the sink PIM managers are also created for sending the attributes of entities. In the matching part, the sink PIM managers send the data of subscription region to the source PIM managers via multicast mode. The source PIM managers perform the overlap tests according the PIM algorithm. In the update part, according to the matching results, 
the source PIM managers send the attributes of matched entities to the sink PIM managers via the point-to-point communication channels. The details of protocol are listed as follows:

1. Initialization

a. Each type of update-subscription region pair is allotted an exclusive multicast group according to a common initial configure file.

b. The PIM managers of the federates check whether the multicast groups are created according to the update/subscription ability of the federates. If the multicast groups are created, the PIM managers join the multicast groups. Otherwise, the PIM managers create and join the multicast groups.

c. The PIM managers send their IP/port addresses and their roles (source or sink, or both) to the multicast group.

d. In case a source/sink PIM manager receives the message of a sink/source PIM manager's $\mathrm{IP} /$ port address and role from the multicast group, they negotiate with each other to create a singular point-to-point communication channel between them for updating the attributes of the entities.

2. Matching

a. Once the subscription federate registers an entity of one object class, the sink PIM managers send the entity handle and the IP/port address to the multicast group which is associated with the subscription region of the entity.

b. Once received the entity handle and the IP/port address from the multicast group, the source PIM managers maintain a subscription entity handle-IP/port map so that it can quickly find the sink PIM manager through the handle of the subscription entity.

c. In case the subscription region of an entity is changed, the sink PIM manager send the new data of the subscription region (i.e. the time interval, the current position of the region, the ranges of the region, the acceleration of the entity, the velocity of the entity) to the multicast group.

d. After the source PIM managers obtained the new data of the subscription region, they perform the matching tests according to the PIM algorithm to find out when and which update regions are overlapped with the subscription region.

3. Update

a. The source PIM managers record the update-subscription entity pairs and overlap time intervals of their regions.

b. When the simulation time advances to the overlap time interval(s) of the entities' regions, the source PIM managers find the sink PIM managers according to the subscription entity handle-IP/port map and send the attributes of the update entities to the sink PIM managers via their communication channel.

c. Once the sink PIM managers receive the attributes of the update entities, they transfer the attributes of the update entities to the subscription federates.

This is the additional cost of the PIM algorithm. The runtime performance improved by IM algorithm is affected by many facets, such as predictive time intervals of entities, the complexity of attributes of entities and the frequency of attribute update.

In contrast to the distributed pattern, the centralized pattern is more suitable for a client-server DVE. In the client-server mode, all models are hosted on a server process. The server manages and updates attributes (e.g. the physical information, such as the position, the direction, 
etc.) so that the clients participating in the DVE can share virtual space. The time consumed by collecting entities' attributes in a computing process is very tiny. Therefore, the PIM algorithm can work well on the single computational node server because it can easily obtain all region information of entities. To HLA-based distributed simulations, implementation of the PIM algorithm in centralized pattern causes that changes to regions must be sent to the Central RTI Component (CRC) from every federate. And then, CRC must send the attributes of entities to the relative federates according to the matching results. This causes substantial network traffic, as well as two network latencies.

According to the analysis of the distributed pattern and the centralized pattern, we implement the PIM algorithm with distributed pattern on KD-HLA/RTI distributed infrastructure [44].

\section{Experiments and results}

Four sets of experiments are conducted to evaluate different aspects of the four IM algorithms, namely:

(1) Frequent interest matching using sort-based algorithm (FIM)

(2) Space-time interest matching using sort-based algorithm (STIM)

(3) Predictive interest matching algorithm using the brute force algorithm (PIM-BF)

(4) Predictive interest matching algorithm using the region pruning method (PIM)

The FIM algorithm divides a simulation timestep into several small time subintervals. At each time subinterval, FIM uses the sort-based algorithm to perform interest matching for all update-subscription region pairs to capture the events between two consecutive timesteps. Its ability to capture missing events depends on the size of the time subintervals. The STIM algorithm is a continuous matching algorithm, which is proposed by Liu and Theodoropoulos [2]. It uses the sweep volume method to cull out the region pairs that are unlikely to overlap with each other. It then implements the divide-and-conquer algorithm to determine the missing events. Similar to the FIM algorithm, the STIM algorithm uses the time threshold to determine whether the region pair overlaps with each other. The PIM-BF algorithm uses the prediction of the space-time intersection of regions method presented in Section 3 to compute the overlap of the region pair. It is an accurate and continuous IM algorithm. However, the PIM-BF algorithm performs the selection of region pairs via the brute force algorithm, which is different from the PIM algorithm. Concretely, the PIM-BF algorithm does not use region pruning method to select regions pairs (shown as Lines: 11-17 in algorithm 2). In PIM-BF algorithm, lines: 11-17 of algorithm 2 is substituted with codes that make region pairs from replanRegionSet and the set of update/subscription regions through brute force method. The PIM is similar to the PIM-BF algorithm except for the selection of region pairs. The region pruning method is used to efficiently pick out the region pairs that potentially overlap with each other.

All four algorithms have the ability to capture the missing events. To quantitatively evaluate the four algorithms, four metrics are designed as follows:

(1) Event-capturing ability: To evaluate the ability of algorithms to capture the missing events.

(2) Redundant matching avoidance: To evaluate the ability of algorithms to avoid redundant matching.

(3) Runtime performance: To evaluate the computational performance of the interest matching process.

(4) Scalability: To evaluate the scalability of IM algorithm on a real distributed simulation 
infrastructure.

\subsection{Experiment setup}

To evaluate the four aspects of these four algorithms, we conducted a real-like test-bed simulation. The simulation uses a World War II dogfight scenario (aerial combat between fighter aircrafts), which has been used as the benchmark by the STIM algorithm [2]. We modified the scenario to make it represent more general situations. The scenario consists of a three-dimension virtual space and a number of agents (aircraft). Agents are equally divided into two teams, which engage each other in an aerial combat. Each agent is associated with an update region and a subscription region. The agents move in the three-dimension virtual space according to their motion functions and predictive time intervals. Some of the essential experimental parameters are listed in table 3. The purpose of advancing the logical time in uniform steps is for the simplification of experiment description and the implementation of the FIM algorithm. The PIM algorithm and the STIM algorithm do not need operate in uniform steps. The STIM, PIM-BF, and PIM algorithms take source filter mode while the FIM algorithm can only take destination filter mode.

For the sake of simplification, no aircraft would be destroyed during the simulation. Distributed pattern is chosen to implement all algorithms in KD-HLA/RTI [44], which is compliant with DMSO 1.3 and IEEE 1516 standards. All simulations were run on 4 PCs. Each PC has a 4-GB main memory and an Intel Core i7-3520 M CPU $(2.9 \mathrm{GHz})$. If there is no specific explanation, agents of each team are equally distributed into two federates. There are four federates in the experimental federations. All experimental results have been obtained as the average over ten runs.

Table 3. Experimental configuration

\begin{tabular}{|c|c|c|}
\hline $\begin{array}{l}\text { Size of Virtual } \\
\text { Space }\end{array}$ & Width $\times$ Length $\times$ High & $\begin{array}{l}\text { The size of virtual space Width } \times \text { Length } \times \text { High is set as } \\
160 \mathrm{~km} \times 160 \mathrm{~km} \times 20 \mathrm{~km} \text {. }\end{array}$ \\
\hline $\begin{array}{l}\text { Number of } \\
\text { Agents }\end{array}$ & $S$ & $\begin{array}{l}\text { The number of agents is denoted by } S \text {. Each agent is } \\
\text { associated with an update region and a subscription region. } \\
\text { The initial positions of agents are uniformly distributed in the } \\
\text { virtual space. }\end{array}$ \\
\hline $\begin{array}{l}\text { Agent } \\
\text { Movement }\end{array}$ & & $\begin{array}{l}\text { The length of the time interval for predicting for each agent is } \\
\text { a random variable. The movements of agents sequentially } \\
\text { changes in the following four modes: } \\
\text {-Search: The agent moves at a quarter of maximum speed to } \\
\text { search targets. If a target appears, it then changes to engage } \\
\text { mode. Under this mode, it turns to a random new direction for } \\
\text { a random time interval between } 60 \text { seconds and } 120 \text { seconds. } \\
\text {-Engage: The aircraft accelerates to the maximum speed and } \\
\text { chases its target for a random time interval between } 120 \\
\text { seconds and } 180 \text { seconds. It then changes to cool mode. } \\
\text {-Cool: The aircraft gradually reduces its speed to a quarter of } \\
\text { maximum speed and the flies for } 1 \text { minute. It then changes } \\
\text { the search mode. In addition, the aircraft cannot engage any } \\
\text { target in this mode but can be engaged by an enemy. } \\
\text {-Evade: If an aircraft is being engaged, it accelerates to the } \\
\text { maximum speed and turns to a new random direction for } \\
\text { every } 5 \text { seconds until it is no longer being engaged. Then, it } \\
\text { changes to the cool mode. }\end{array}$ \\
\hline $\begin{array}{l}\text { Maximum } \\
\text { Speed of Agent }\end{array}$ & $m v$ & $\begin{array}{l}\text { The maximum speed of the agent is represented as a random } \\
\text { variable, which is subject to uniform distribution at } \\
\text { boundaries of } 800(\mathrm{~km} / \mathrm{L}) \text { and } 1200(\mathrm{~km} / \mathrm{L}) \text {. The initial speed } \\
\text { is } m v / 4 \text {. }\end{array}$ \\
\hline $\begin{array}{l}\text { Acceleration } \\
\text { of Agent }\end{array}$ & $a$ & $\begin{array}{l}\text { The acceleration of the agent is represented as a random } \\
\text { variable, which follows uniform distribution at boundaries of } \\
5 \mathrm{~m} \cdot \mathrm{s}^{-2} \text { and } 7 \mathrm{~m} \cdot \mathrm{s}^{-2} \text {. The gravitational acceleration is set } \\
\text { as } 9.8 \mathrm{~m} \cdot \mathrm{s}^{-2} \text {. }\end{array}$ \\
\hline
\end{tabular}




\begin{tabular}{|c|c|c|}
\hline Update Region & $U W \times U L$ & $15 \mathrm{~m} \times 15 \mathrm{~m} \times 10 \mathrm{~m}$ \\
\hline $\begin{array}{l}\text { Subscription } \\
\text { Region }\end{array}$ & $S W \times S L$ & $5 \mathrm{~km} \times 5 \mathrm{~km} \times 3 \mathrm{~km}$ \\
\hline $\begin{array}{l}\text { Timestep of } \\
\text { Simulation }\end{array}$ & $\Delta t$ & $\begin{array}{l}\text { For the sake of simplification, simulation time is designed to } \\
\text { advance in uniform steps. The timestep is denoted by } \Delta t \text { and } \\
\text { is set as one second in simulation time. }\end{array}$ \\
\hline $\begin{array}{l}\text { Execution } \\
\text { Time of } \\
\text { Simulation }\end{array}$ & ETS & $\begin{array}{l}\text { Average execution time of the matching algorithms is } \\
\text { measured over } 5,000 \text { timesteps. }\end{array}$ \\
\hline $\begin{array}{l}\text { Count of } \\
\text { Overlaps }\end{array}$ & $C O$ & $\begin{array}{l}\text { The total count of overlaps reported by matching algorithms } \\
\text { in the whole simulation execution. The count is the integer } \\
\text { part of average value of ten runs. }\end{array}$ \\
\hline $\begin{array}{l}\text { Time interval } \\
\text { of FIM }\end{array}$ & $\sigma t$ & The time interval of the FIM to perform matching operations. \\
\hline $\begin{array}{l}\text { Time } \\
\text { Threshold of } \\
\text { STIM }\end{array}$ & $\delta t$ & $\begin{array}{l}\text { The time threshold of the STIM to test whether to split the } \\
\text { time interval into two equal subintervals. }\end{array}$ \\
\hline
\end{tabular}

6.2 Event-capturing ability

The first set of experiments compares FIM, STIM, PIM-BF, and PIM for their ability to capture the missing events with the number of agent entities extending from 100 to 1600 . To do so, we execute the experiments with 5000 timesteps and count the total number of region overlaps detected by the four algorithms. The time interval $\sigma t$ of FIM and the time threshold $\delta t$ of STIM are both set as $\Delta t / 64$.

The overlap count of the four algorithms is illustrated in Fig. 7. We find that when the number of agents increases, the overlap count of all algorithms also increases. The reason for this phenomenon is when there are more agents in the virtual space, the chance of region overlap increases.

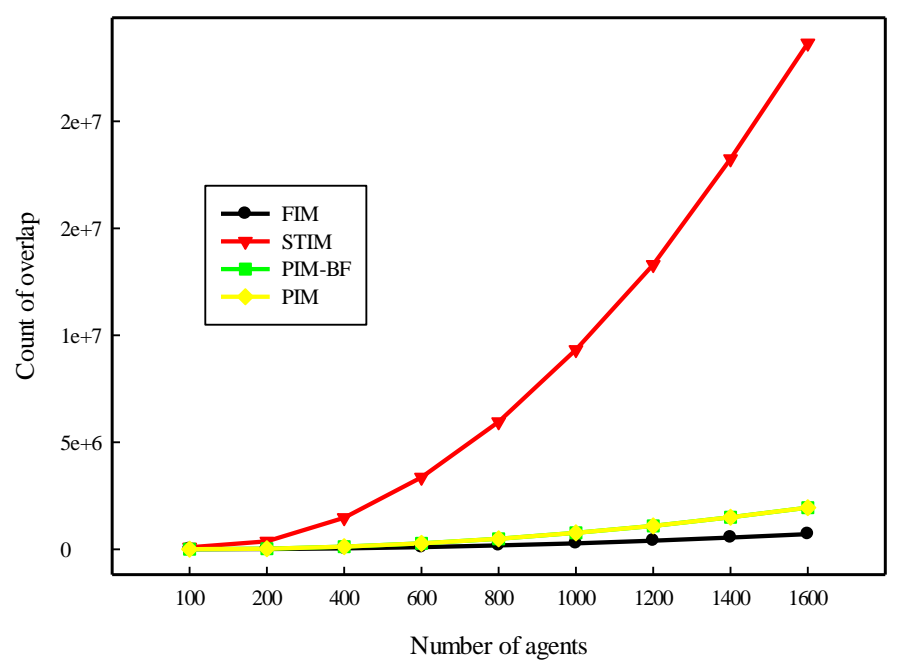

Fig. 7 Event-capturing ability of four IM algorithms

Another observation is that PIM-BF and PIM have the same overlap count under the same number of agents because both of these two algorithms use the prediction of the space-time intersection of regions method to detect the region overlap. Their event-capturing ability should be the same. Most importantly, the results show that the number of overlaps detected by STIM is more than that of PIM, and the difference becomes significant when the number of agents increases. As mentioned in previous sections, PIM detects the region overlap by solving the motion functions of agents. Therefore, the number of region overlaps obtained by PIM is accurate. However, STIM uses the axis-aligned swept volume (AASV) of regions other than the region to 
detect region overlap, which can cause the spurious events. Even two regions do not actually overlap with each other, and STIM considers these two regions overlap if their AASV overlap. Thus, the overlap count detected by STIM is greater than that of PIM. Moreover, when the number of agents increases in the virtual space, the probability of AASV overlap is greater than the chance of region overlap. This also explains that the number of overlaps detected by STIM increases more sharply than that of PIM.

The results in Fig. 7 also show that the number of overlaps detected by FIM is less than that of PIM. As mentioned previously, FIM only performs interest matching at discrete points of time. It is not able to detect the overlaps happening between discrete time subintervals. Many overlaps are ignored by discrete algorithms, while the PIM algorithm can still capture them. Consequently, its event-capturing ability is weaker than PIM.

For FIM, one simple method to improve the event-capturing ability is to reduce the time interval of matching and perform frequent discrete interest matching. To do so, we reduce the time interval $\sigma t$ of FIM from $\Delta t / 2$ to $\Delta t / 128$ and compare the results with PIM. In this experiment, the number of agents is fixed at 1000. The result is shown in Fig. 8. We find that more overlaps can be captured by FIM when the time interval decreases. However, when the time interval $\sigma t$ is reduced to $\Delta t / 64$, the number of overlaps becomes stable. In the meantime, the count of overlaps captured by PIM remains the same. We can conclude that although more overlaps can be captured by FIM by reducing its time interval, there are still many overlaps occurring between two consecutive matching operations of FIM, which are missed by FIM. The difference in overlap count between FIM and PIM could be regarded as the "missing events."

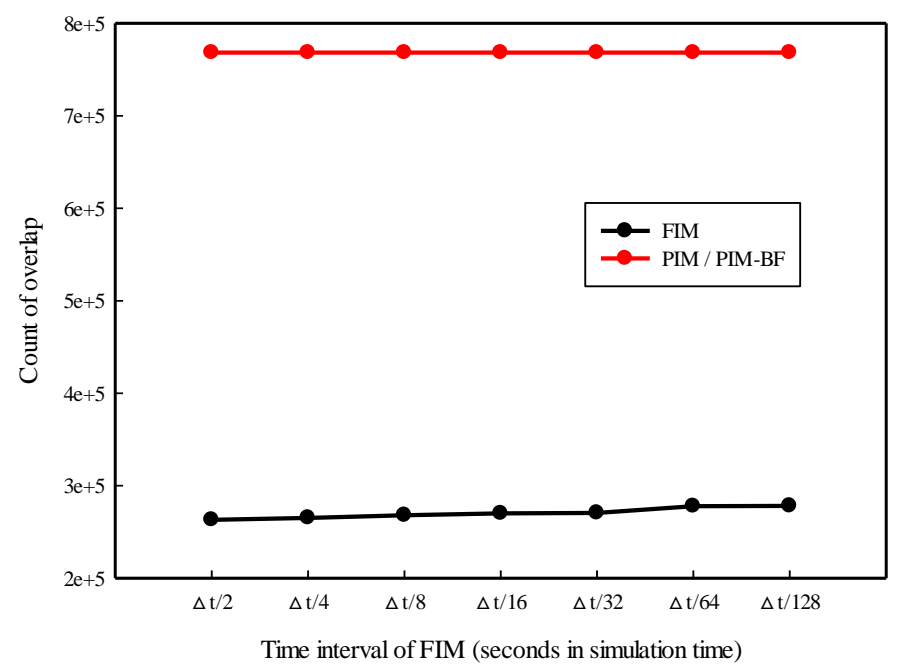

Fig. 8 Event-capturing ability of the IM algorithms

As for STIM, the method to reduce the "spurious events" is to decrease the time threshold. To compare the accurate event-capturing ability of STIM with that of PIM, we extend the time threshold $\delta t$ of STIM from $\Delta t / 2$ to $\Delta t / 128$ in this experiment. The experimental results are shown in Fig. 9. The results indicate that when the value of the threshold decreases, the overlap count of the STIM algorithm gradually drops because decreasing the threshold would cause the algorithm to perform more recursions and thus would increase the filtering precision of the algorithm. When the overlap count of STIM reaches a steady number $(\approx 9,323,501)$, the number is approximately twelve times the result of PIM $(\approx 768,369)$. The results reveal that although STIM 
can capture the "missing events", it introduces the "spurious events". Its filtering precision is lower than that of PIM.

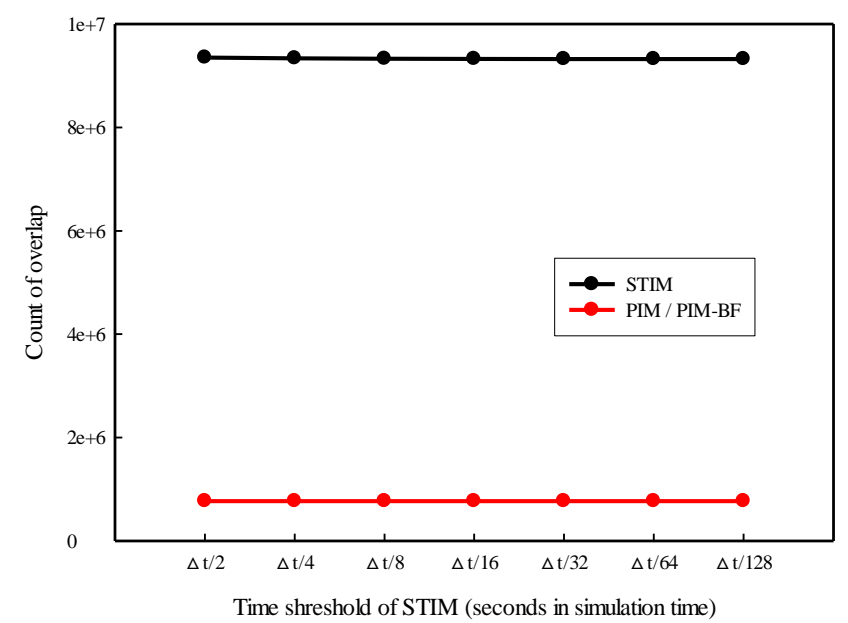

Fig. 9 Event-capturing ability of the IM algorithms

The event-capturing ability of IM algorithms can also be evaluated in terms of precision and recall, which are the metrics used in information retrieval research [43]. When the time interval $\sigma t$ of FIM and the time threshold $\delta t$ of STIM are both set as $\Delta t / 64$, the precision of IM algorithms is shown in Fig. 10 (a). According to algorithm1 and algorithm 2, we know that PIM algorithm and PIM-BF algorithm are accurate IM algorithms. The overlaps reported by them have actually occurred. So, their precisions are both $100 \%$. As for FIM algorithm, each overlap detected by it has also occurred. Therefore, FIM algorithm also has $100 \%$ precision. However, STIM algorithm may report spurious overlap events. Although the precision of STIM algorithm increases when the number of agents increases, the highest precision of STIM algorithm is about $8 \%$. In other word, about $92 \%$ of overlaps reported by STIM algorithm are spurious events.

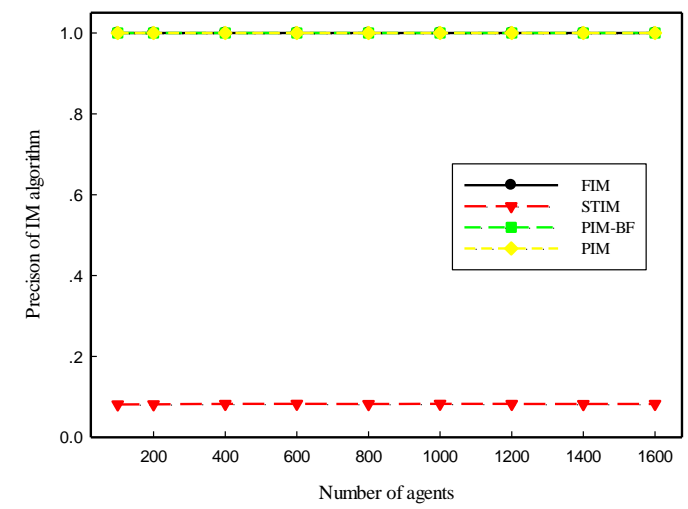

a. precision

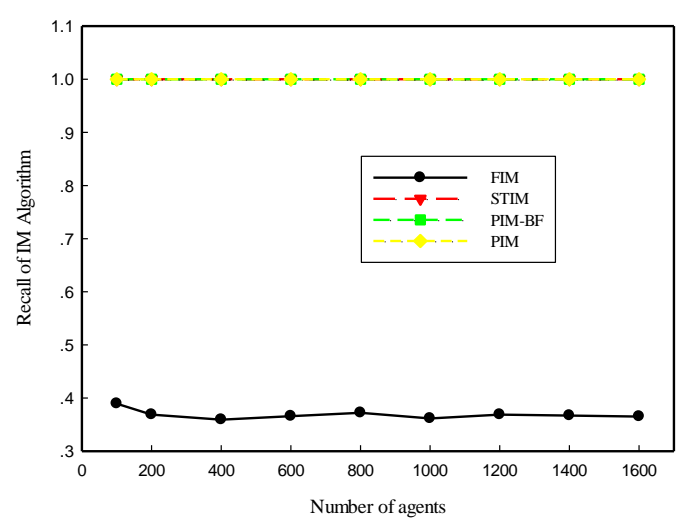

b. recall

Fig. 10 The precision and recall of the IM algorithms (number of agents varying)

The recall of the four IM algorithms is shown in Fig. 10(b). The recall of STIM, PIM-BF and PIM are $100 \%$, which imply that each overlap actually occurred must be reported by those algorithms. However, as mentioned above, FIM algorithm may fail to report events. Therefore, the recall of FIM can not be $100 \%$. When the number of agent increases, the recall of FIM algorithm decreases and becomes stable (about $81 \%$ ). 
For FIM, reducing the time interval of matching can improve its event-capturing ability. Therefore, the recall of FIM will increase when time interval of matching decreases, which is shown in Fig. 11. We find that the increment of recall is not linear relation to the decrement of time interval. When the time interval decreases, the recall of FIM algorithm increases and becomes stable (about 36\%).

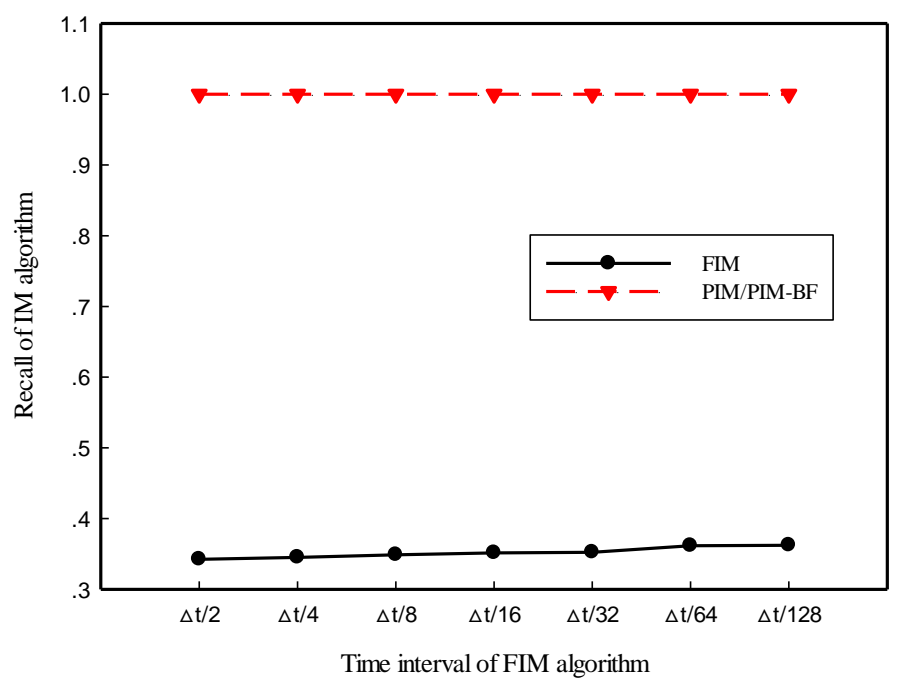

Fig. 11 The recall of IM algorithms

As for STIM, reducing the time interval of matching will increase its precision. Fig. 12 shows that the precision of STIM increases when the time interval decreases. However, the increment of precision is not linear relation to the decrement of time interval. When the time interval decreases, the precision of STIM algorithm increases and becomes stable (about 8.24\%).

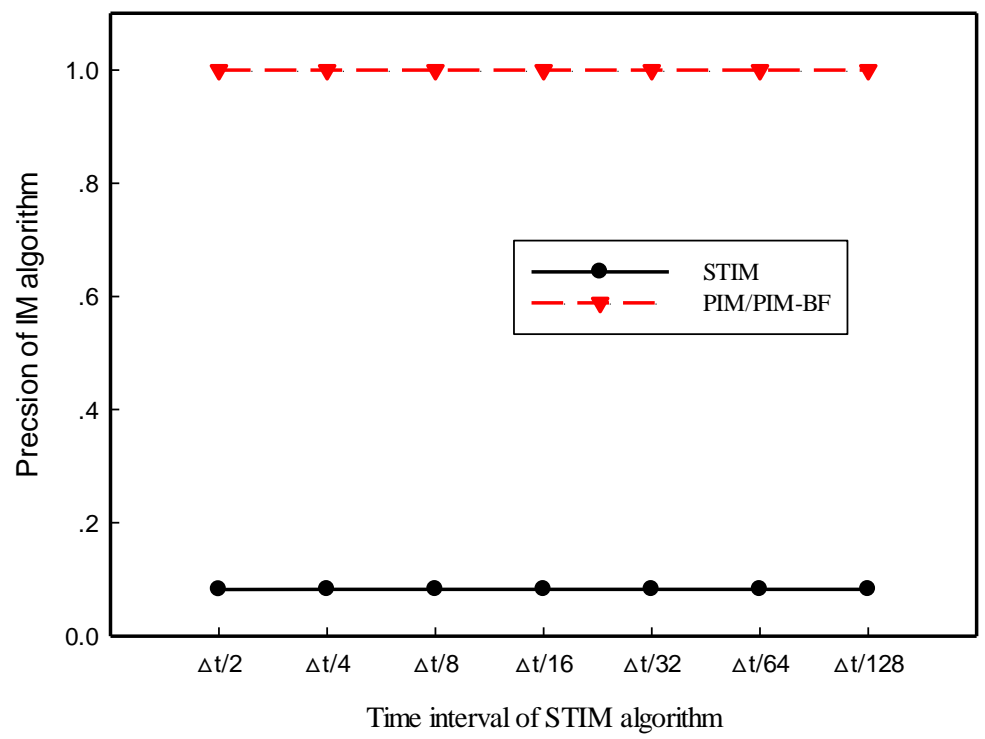

Fig. 12 The precision of the IM algorithms

6.3 Redundant matching avoidance

The PIM algorithm does not perform overlap tests of regions at discrete timesteps. At the beginning of the predictive time interval, our algorithm can exactly predict the overlap time 
intervals of regions. For example, in Fig. 5, assuming the simulation timestep is one, the discrete IM algorithms should perform matching at every timestep to obtain the overlap of two regions. However, the PIM algorithm only needs to compute the ROTI of two regions once at the beginning of the predictive time interval. Compared with the discrete IM algorithms, nineteen times matching can be avoided in our algorithm, which can drastically improve the runtime performance of the algorithm.

The second set of experiments compares FIM, STIM, PIM-BF, and PIM for their ability to avoid redundant matching with the number of agents extending from 100 to 1600 . We execute the experiments with 5000 timesteps and count the total number of region overlaps and the count of matching performed by the four algorithms. The time interval $\sigma t$ of FIM and the time threshold $\delta t$ of STIM are both set as $\Delta t / 64$ again.

The experimental results are shown in Fig. 13 and Table 4. To capture the events, the matching count of FIM is the largest in the four algorithms. The redundant matching count grows exponentially with the number of agents. The second poorest algorithm is the STIM. The STIM uses the swept volume to capture missing events. It certainly causes a large count of redundant matching.

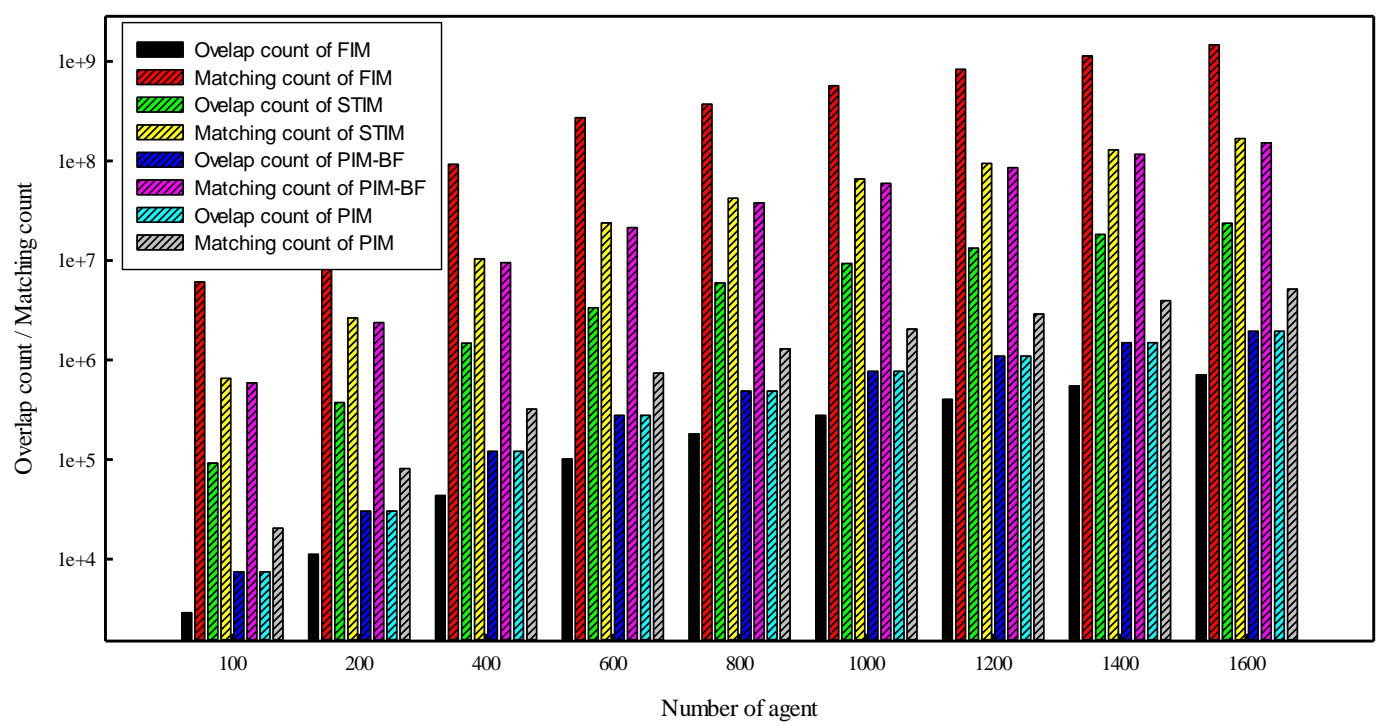

Fig. 13 Redundant matching avoidance of the IM algorithms

Table 4. Matching count and overlap count of the IM algorithms

\begin{tabular}{|l|r|r|r|r|r|r|r|r|r|}
\hline Number of agents & 100 & 200 & 400 & 600 & 800 & 1000 & 1200 & 1400 & 1600 \\
\hline Overlap count of FIM & 2908 & 11206 & 43491 & 101524 & 181421 & 277758 & 403122 & 548243 & 710158 \\
\hline Matching count of FIM & 6092410 & 23694685 & 92335390 & 271804520 & 370393750 & 570083873 & 830219428 & 1134206291 & 1465685920 \\
\hline Overlap count of STIM & 92133 & 373796 & 1472264 & 3362316 & 5956051 & 9323927 & 13306183 & 18245156 & 23654582 \\
\hline Matching count of STIM & 652341 & 2644789 & 10415265 & 23797597 & 42169622 & 66004436 & 94207390 & 129168821 & 167447601 \\
\hline Overlap count of PIM-BF & 7468 & 30383 & 121059 & 277500 & 487490 & 768369 & 1093496 & 1493783 & 1944997 \\
\hline Matching count of PIM-BF & 588807 & 2375041 & 9538985 & 21445947 & 38006733 & 59469311 & 85587825 & 116408057 & 152169215 \\
\hline Overlap count of PIM & 7468 & 30383 & 121059 & 277500 & 487490 & 768369 & 1093496 & 1493783 & 1944997 \\
\hline Matching count of PIM & 20473 & 81326 & 321888 & 738642 & 1295493 & 2041652 & 2902756 & 3952546 & 5161419 \\
\hline
\end{tabular}

Because both STIM and PIM use similar methods to cull out the region pairs that are unlikely to overlap with each other, both STIM and PIM are better than FIM. In addition, these two 
algorithms have a similar ability to avoid redundant matching.

The computation swept volume of a region in STIM is based on the time threshold $\delta t$, and the computation of the swept volume of a region in PIM is based on the predictive time interval. Generally, the size of the swept volume of regions in PIM is larger than that in STIM. It is expected that the redundant matching count of PIM should be greater than that of STIM. However, the count of the swept volume computation of PIM is less than that of STIM. The matching count of PIM is actually less than that of STIM. So, PIM has a better ability to avoid redundant matching than STIM.

6.4 Runtime performance

We conduct the third set of experiments and compare FIM, STIM, PIM-BF, and PIM for their runtime performance with the number of agents extending from 100 to 1600 . The execution time of the four algorithms is the average wall-clock time consumed at each simulation timestep. The time interval $\sigma t$ of FIM and the time threshold $\delta t$ of STIM are both set as $\Delta t / 64$ again.

The execution time of the four algorithms is shown in Fig. 14. The results illustrate that when the number of agents increases, the execution time of all algorithms also increases. This is due to the increase in the number of region pair matching operations of all algorithms when more agents are added to the virtual space.

Another observation from the results is that although PIM-BF performs interest matching by using the brute force algorithm and FIM uses the sort-based algorithm to perform interest matching, the FIM algorithm consumes more computational time than PIM-BF. This difference becomes significant when the number of agents is gradually increased. This is due to two reasons. The first reason is the overhead introduced by sorting all of the end points in the range of regions in the FIM algorithm. The second reason is that the PIM-BF algorithm only tests the regions whose motion function is updated.

Figure 15 also shows that the STIM algorithm has better runtime performance than FIM and PIM-BF. Although both STIM and FIM perform interest matching with the sort-based algorithm, STIM use the swept volume to cull out region pairs that are unlikely to overlap with each other. As mentioned in previous sections, STIM has a better ability to avoid redundant matching. Therefore, STIM requires less computational effort than FIM. Similarly, although the prediction of the space-time intersection of the region method used by PIM-BF is more efficient than the divide-and-conquer algorithm used by STIM, the count of redundant matching for PIM-BF is considerably greater than that of STIM. So, PIM-BF has a poorer runtime performance than STIM.

A more important finding in Fig. 14 is that the PIM algorithm has better runtime efficiency than the other three algorithms. Although both PIM and STIM use swept volumes to avoid unnecessary region pair matching, the count of redundant matching for PIM is less than that of STIM (see Table 4). Moreover, the overhead introduced by the divide-and-conquer algorithm in STIM is also greater than the time consumed by the intersection computation of region pairs in PIM. Therefore, PIM requires less computational effort to perform interest matching than STIM.

Although both PIM and PIM-BF use the prediction of the space-time intersection of regions method to perform interest matching, PIM uses the region pruning method to reduce redundant matching greatly. PIM certainly performs better than PIM-BF. For PIM and FIM, although there is some overhead introduced by the computation of swept volumes and the intersection of region pairs in PIM, the count of redundant matching for FIM is considerably greater than that of PIM. 
Redundant matching decreases the performance of FIM and makes it perform worse than PIM.

For the FIM algorithm, the time interval is a main parameter that affects its runtime performance. Although decreasing the time interval of FIM can improve its event-capturing capability, the performance of the FIM algorithm is also degraded. We conduct an experiment to compare the runtime efficiency of FIM, PIM-BF and PIM with the time interval $\sigma t$ of FIM extending from $\Delta t / 2$ to $\Delta t / 128$. In this experiment, we set the number of agents to a constant 1200 again.

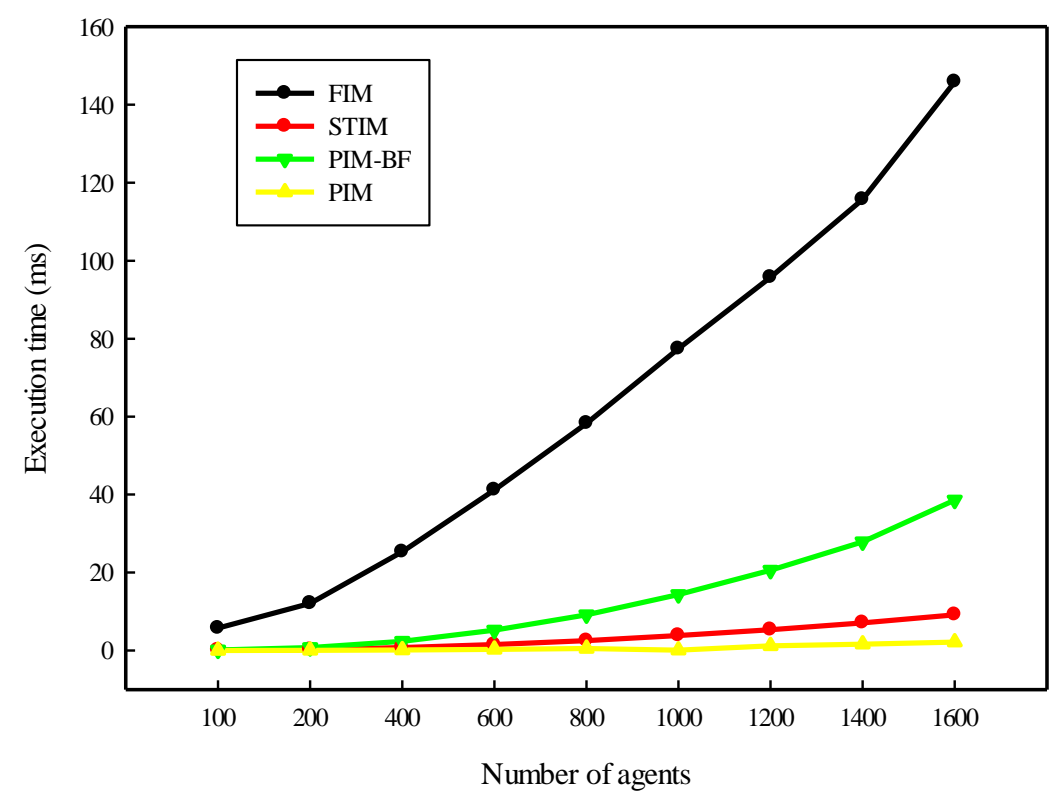

Fig. 14 Execution time of four IM algorithms

Figure 15 shows the execution time of FIM, PIM-BF and PIM. We observe that the execution time of FIM increases quickly when its time interval is decreased. This is caused by the extra computational effort required by FIM to perform overlap evaluations for all regions at each discrete point of time. The computational overhead of FIM is negative proportional to the size of its time interval. The execution time of PIM-BF and PIM remains the same as the time interval varies. Another observation is that when the time interval of FIM is greater than $\Delta t / 16$, PIM-BF performs better than FIM. More importantly, with all values of the time interval, PIM consumes less time to capture all missing events than FIM.

For the STIM algorithm, as mentioned in the previous section, reducing the time threshold can improve its filtering precision. However, the runtime performance of STIM can also be affected by the time threshold. To clarify the relationship between the time threshold of FIM and its runtime efficiency, we extend the time threshold $\delta t$ of STIM from $\Delta t / 2$ to $\Delta t / 128$ in this experiment. The number of agents is again set as a constant 1200 .

Figure 16 shows the execution time of STIM, PIM-BF and PIM. We notice that the change of execution time of STIM with different values of time thresholds is very tiny and can be ignored. The STIM algorithm uses the divide-and-conquer method to divide timesteps into time subintervals to perform overlap tests for region pairs. Once it detects that the two regions overlap with each other, it ends the process for the overlap test and ignores the remnant of time subintervals. Therefore, the STIM algorithm does not test overlap states for a region pair at all 
time slices. Decreasing the time interval of STIM does not introduce more computational overhead.

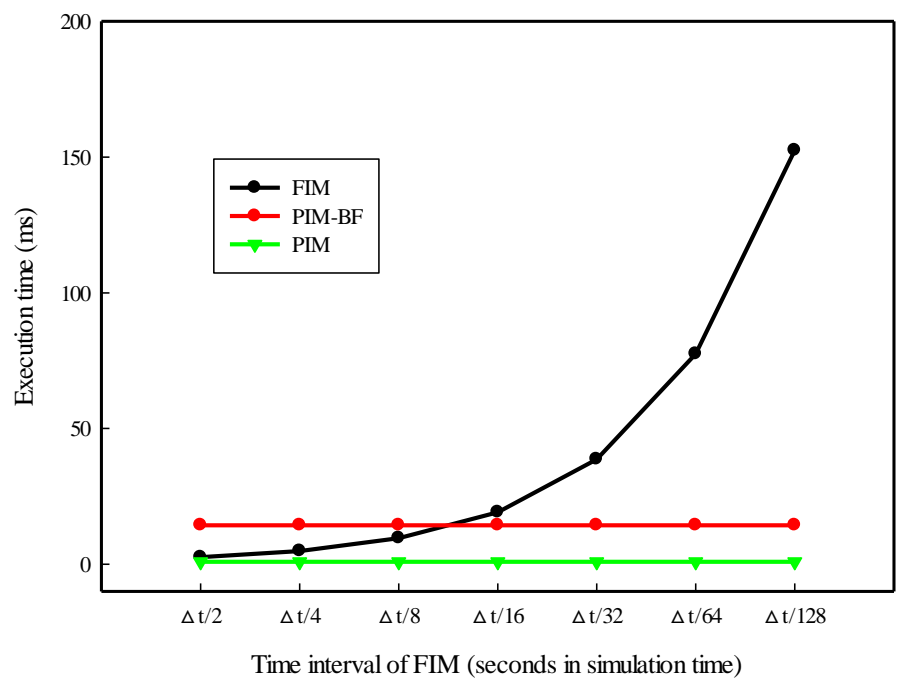

Fig. 15 Execution time of FIM, PIM-BF and PIM

We also note that the PIM-BF algorithm uses more execution time than the STIM algorithm. This is due to the brute force algorithm introduced by PIM-BF. Many unnecessary comparisons are performed to capture events. For the PIM algorithm, its performance is better than that of the STIM algorithm. PIM uses the prediction method of the space-time intersection to avoid more redundant matching compared with STIM. The overhead of swept volumes for PIM is also slighter than that of STIM.

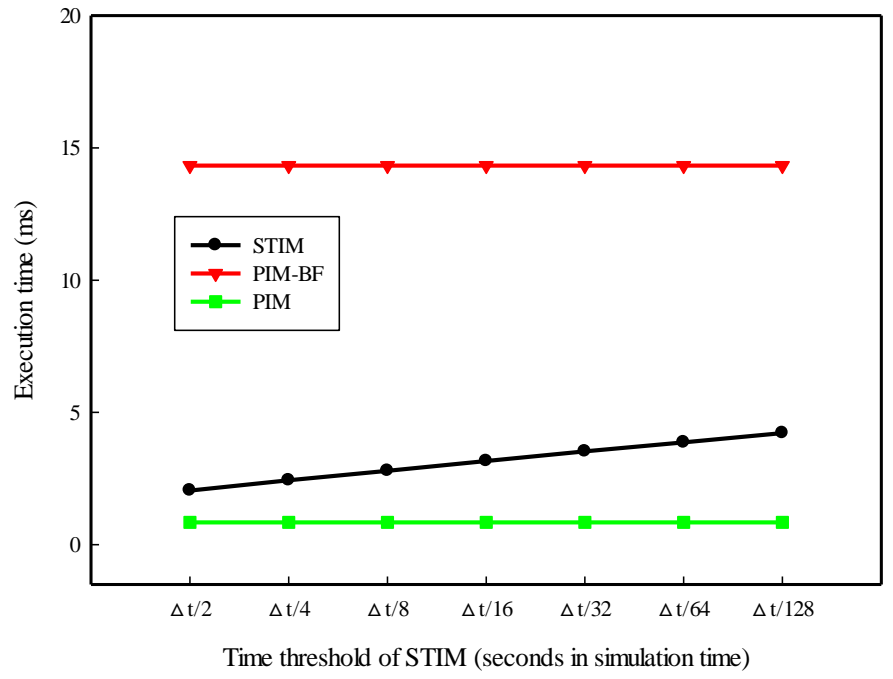

Fig. 16 Execution time of STIM, PIM-BF and PIM

\subsection{Scalability}

In the fourth set experiments, we compare FIM, STIM, PIM-BF, and PIM on their scalability and time overhead. Firstly, the number of federates is set as 4 and the number of agents extends from 100 to 1600 . Then, the number of agents is fixed to 1000 and the number of federate extends from 2 to 10. Agents are uniformly distributed on federates. And, federates are uniformly hosted on computing nodes. The time interval $\sigma t$ of FIM is set as $\Delta t / 2$ and the time threshold $\delta t$ of 
STIM is set as $\Delta t / 64$.

To detect overlaps of regions, STIM, PIM-BF, and PIM algorithms need to collect all predictive time intervals, update regions, subscription regions and movement polynomials of entities distributed on different federates. Therefore, while these attributes of agents change, these three IM algorithms on a federate must update these attributes to other federates, which is called information update. Information update for STIM, PIM-BF, and PIM algorithms includes updating data of predictive time intervals, update regions, subscription regions and movement polynomials of entities. As for FIM, information update consists of updating of regions (including update region and subscription region).

The average count of information update for IM algorithms on each federate is shown in Fig. 17. The count of information update for STIM, PIM-BF, and PIM algorithms is determined by the motion dynamics of agents and the number of agents on a federate. Therefore, their counts of information collection are the same. However, the count of information update for FIM algorithm is governed by the time interval $\sigma t$ of FIM and the number of agents on a federate. The count of information update of FIM algorithm is much larger than that of other three IM algorithms (about 1000 times as shown in Fig. 17 (a)). When the number of agent is fixed and the number of federates increases, the counts of information update of four IM algorithms on each federation decrease.

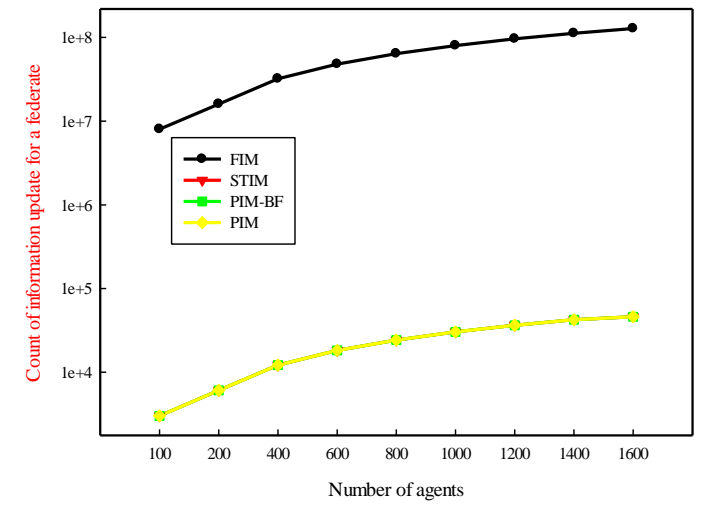

a. Number of agents varies

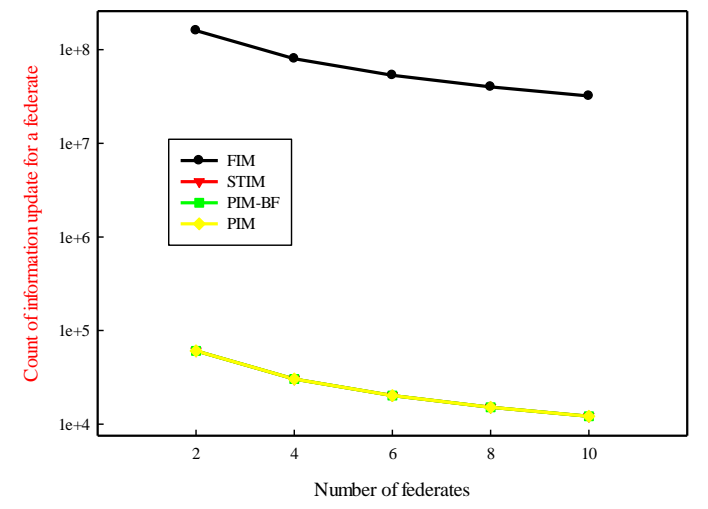

b. Number of federates varies

Fig. 17 Count of information collection of the IM algorithms

The time overhead of IM algorithms consists of time spent on information update and time consumed by algorithm execution. The time overhead of four IM algorithms is shown in Fig. 18, which is wall-clock time spent on 5000 steps execution of federation. Compared with the results in Fig. 14, we conclude that time spent on information update is the main part of time overhead of an IM algorithm. As shown in Fig. 18 (a), the distinction of time overhead of STIM, PIM-BF, and PIM algorithms are small when the number of agents is less than 600 . We find that when the number of agents increases, the time overhead of four IM algorithms also increases and the distinction of time overhead of STIM, PIM-BF, and PIM algorithms increases. The time overhead of the PIM algorithm is the smallest. Another observation, as shown in Fig. 18 (b), is that time overhead of IM algorithms on each federate decreases when the number of federates increases.

The counts of attribute update of agents on a federate based on matching result of IM algorithms are shown in Fig. 19. We find that the count of attribute update based the FIM algorithm is directly proportional to the number of agents on a federate, as shown in Fig. 19 (a). 
This phenomenon is caused by the reason that the FIM algorithm needs to collect regions of all agents in the federation at every simulation step. We also find that the count of attribute update performed by the STIM algorithm is large than that of PIM-BF and PIM algorithm, and the difference becomes significant when the number of agents increases. The reason for this phenomenon is that PIM detects the region overlap by solving the motion functions of agents. Therefore, the number of region overlaps obtained by PIM is accurate. However, STIM uses the axis-aligned swept volume (AASV) of regions other than the region to detect region overlap, which can cause the spurious events.

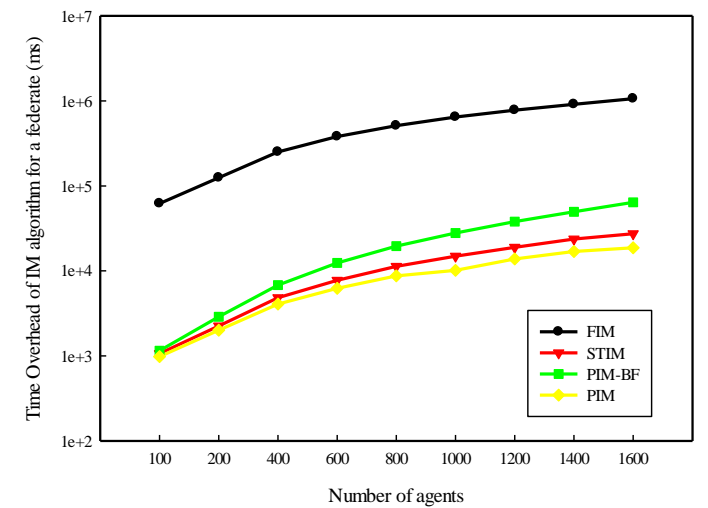

a. Number of agents varies

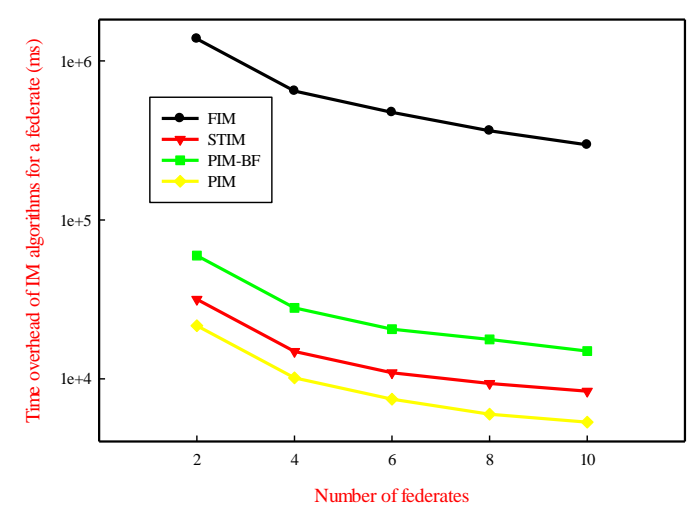

b. Number of federates varies

Fig. 18 Time overhead of the IM algorithms

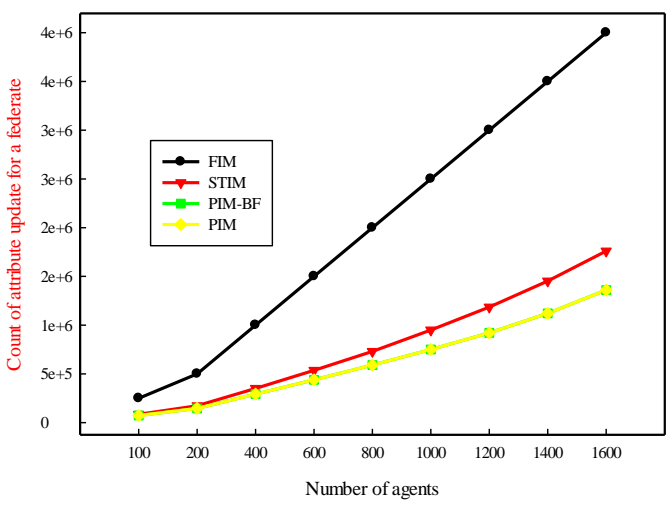

a. Number of agents varies

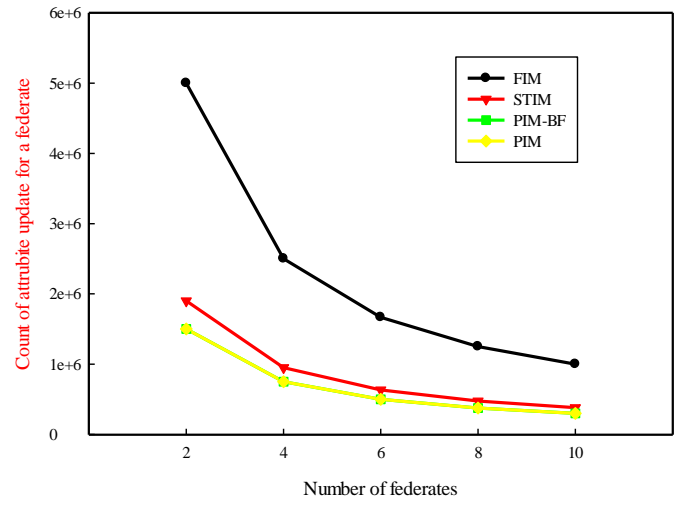

b. Number of federates varies

Fig. 19 Count of attribute update of a federate for IM algorithms

The execution time of a federate is shown in Fig. 20. The results illustrate that when the number of agent increases, the execution time of federates based on four IM algorithms also increases. This is due to the increase in the time overhead of IM algorithms, and the time consumed by agent attribute update and model computing. We find that the execution time of the federate based the FIM algorithm is directly proportional to the number of agents on a federate, as shown in Fig. 20 (a). This is caused by the reason that the FIM algorithm does not reduce the count of attribute update. Although the STIM algorithm is more efficient than the PIM-BF algorithm (as shown in Fig. 14), the federate based on the STIM algorithm executes more slowly than the one based on the PIM-BF algorithm. This phenomenon is caused by the reason that the federate based on the STIM algorithm updates more irrelative attributes to the destination 
federates than the federate based on the PIM-BF algorithm (as shown in Fig. 19 (a)). Another observation is that the execution time of a federate with the PIM algorithm is the most efficient and scalable in the four algorithms.

Figure 21 (b) shows the execution time of a federate with different IM algorithms decreases when the number of federates in a federation increases. Compared with the situation of 2 federates, the speedup of FIM, STIM, PIM-BF and PIM algorithm in the situation of 10 federates are about $2.31,3.49,3.88,4.12$, respectively. Therefore, the PIM algorithm is the most scalable in the four IM algorithms.

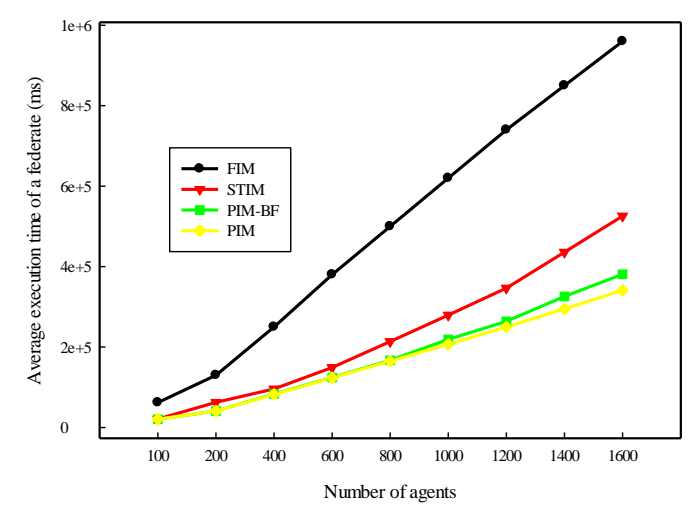

a. Number of agents varies

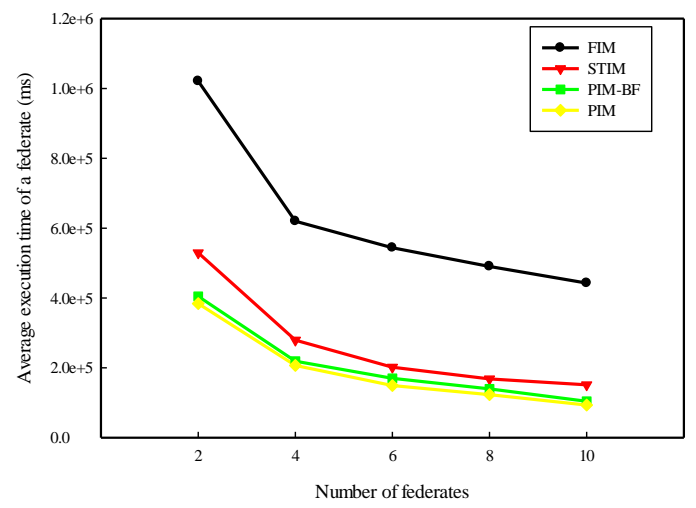

b. Number of federates varies

Fig. 20 Average execution time of a federate

\section{Issues for discussion}

\subsection{Applicability}

Within Modeling and Simulation (M\&S) community, many researchers use different terms, such as multiple paradigms, formalisms, or multi-resolution modeling, to describe models and simulation applications [54-58]. A taxonomy is proposed by Sulistio for classifying terminologies that describe simulations with multiple models [59]. The taxonomy provides a mutually exclusive description for simulation models based on the characteristics of time representation, behavior, execution, expression, and resolution [60]. Although there is not a strict taxonomy for low resolution applications and high resolution applications, many researchers, especially in defense M\&S community, reach a common consensus that: high resolution simulations are entity level simulations where singular objects, e.g. soldiers, tanks, aircrafts, are represented with atomic models; low resolution applications, also called highly aggregated simulations, are aggregated level simulations where collections of objects, i.e., units, aircraft formations, tank battalions, are represented with aggregated models [55][61-63]. According to the practices of simulation applications and to the best of our knowledge, the motion models within many of low resolution simulation applications can be described with quadratic polynomials [62-65].

In order to analyze the simulation applications that are suitable for the PIM algorithm, we classify applications into two categories: low resolution applications and high resolution applications. Low resolution applications are those whose motion model can be described with quadratic polynomials. As for high resolution applications, their motion models need be described by more complex functions than quadratic polynomials. For example, traditionally, military simulations require a hierarchical suite of simulation models to address engineering, engagement, 
mission and theater/campaign measures of performance, measures of effectiveness and measures of merit. Generally, engineering, engagement simulations need functions which are more complex than quadratic polynomials to describe the motion of entities. Therefore, engineering, engagement simulations are high resolution applications. Similarly, some networked game applications can be classified into high resolution applications [42][47-53][66-67].

However, mission and theater/campaign simulations, such as JAS (previously called the Joint Warfare System (JWARS)), JTLS (Joint Theater Level Simulation) and other systems [44-46], may not require such high-resolution models as engineering and engagement simulations. Most of those simulation systems are used to evaluate and optimize mission or campaign problems. The entities in those simulation systems are modeled in low-resolution. The tactical actions, such as turning, rolling and climbing of entities are ignored. The interaction of entities (e.g. sensing, engagement) is calculated according to the conditional probability. The conditional probability is generated from the results of engineering and engagement simulations. For example, a fighter plane group of red side engages with a fighter plane group of blue side. The red group just flights to the destination airspace along with some waypoints. Then, the red group engages with the blue group and returns back to its air force base. The movement of plane groups between two waypoints is a straight line, and the velocity of one plane group keeps unchanged. For another example, virtual entities move between hexagonal regions. The movements of entities between two hexagonal regions are also straight lines. The velocity of one entity keeps the same when it moves between two hexagonal regions. The movements of those entities are described by quadratic polynomials. Therefore, the mission and theater/campaign simulations that use quadratic polynomials to describe the motion of entity can be called low resolution applications.

According to the category of simulation applications, the PIM algorithm can be undoubtedly used in the low resolution application. Assuming there is a quadratic polynomial $\vec{Y}_{(t)}$ that describes the movement of the entity in the low resolution application during the time interval $\left[t_{a}, t_{b}\right] \cdot \vec{Y}(t)$ can be expressed as equation (15) in three-dimension Cartesian space:

$$
\left.\vec{Y}(t)=\vec{Y}_{(t}\right)+x_{1}(t) \hat{i}+x_{2}(t) \hat{j}+x_{3}(t) \hat{k}=x(t) \hat{i}+y(t) \hat{j}+z(t) \hat{k},
$$

where $\hat{i}, \hat{j}$ and $\hat{k}$ are the unit vectors and $x, y$ and $z$ are the coordinates of the virtual entity, which vary with time $t$ according to the quadratic polynomials $x_{1}(t), x_{2}(t)$ and $x_{3}(t)$, respectively.

As is shown in the experimental results of this study, in contrast to the more general but less effective/efficient IM algorithms, the PIM algorithm can accurately capture missing-events and reduce the amount of data transmission for optimal bandwidth for these applications.

In high resolution applications, due to their entities' movement cannot be described by quadratic polynomials or their movement behaviors are difficult to be predicted, the PIM algorithm can not be directly applied to those simulations. However, if there is a time-dependent, real-value continuous function ${\overrightarrow{Y^{\prime}}}^{\prime}(t)$ to describe entity dynamics, an $n^{\text {th }}$ order polynomial in the form of a truncated Taylor expansion can be used to predict the value $\vec{Y}^{\prime}(t)$ at time interval $\left[t_{a}, t_{b}\right]$, which is a common solution used in standard Dead-Reckoning of distributed simulations. $\overrightarrow{Y^{\prime}(t)}$ can be expressed as follows.

$$
\overrightarrow{Y^{\prime}(t)}=\sum_{i=0}^{n} \frac{\vec{P}^{(i)}\left(t_{a}\right)}{i !}\left(t-t_{a}\right)^{i}+\delta E,
$$

where $\vec{Y}_{Y^{\prime}}^{(i)}$ is the $i^{\text {th }}$ derivative of ${\overrightarrow{Y^{\prime}(t)}}$ at time $t_{a}$ and $\delta E$ is the estimation error. Higher-order derivatives introduce higher sensitivity to rapid changes of the entity motion and may result in highly jerky approximations. Therefore, the $1^{\text {th }}$ and the $2^{\text {th }}$-order Dead-Reckoning are the most commonly used. 
According to equation (15), $\overrightarrow{Y^{\prime}(t)}$ can be rewritten as follows.

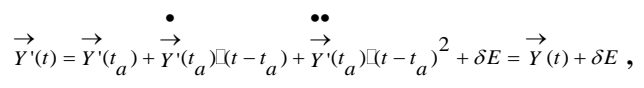

where $\vec{Y}(t)$ is defined in equation (15). Now, the PIM algorithm can be employed to high resolution applications through compensating estimation error $\delta E$ with the region expansion method [15]. Although the region expansion method decreases the precision of the PIM algorithm, which will introduce spurious events, the PIM algorithm is still a proper approach to solve the IM problem of high resolution application. Actually, some IM algorithms have the same assumptions as our algorithm [2][42][48][50][51][53]. Aiming at reducing the actual frequency of attribute update, these algorithms use Dead-Reckoning method based on quadratic polynomials to predict the movement of entities.

In summary, the PIM algorithm not only could be used in low resolution applications, but also be suitable for high resolution applications with the $2^{\text {th }}$-order Dead-Reckoning approach.

\subsection{Analysis of effectiveness}

In the experiments of this study, the PIM algorithm outperforms the FIM algorithm and the STIM algorithm in terms of event-capturing ability, irrelative message reducing, runtime efficiency and scalability.

As shown in experiments mentioned in the above section, the PIM algorithm can capture all events but does not cause spurious events while the event recall of the FIM algorithm is about $34 \%$ and the precision of the STIM algorithm is about $8.2 \%$. Based on its accurate message filtering ability, the PIM algorithm can avoid irrelative messages transmitted between distributed computing nodes. Compared to the STIM algorithm, the PIM algorithm can filter most irrelative messages (about $91.8 \%$ ).

Because the PIM algorithm can filter irrelative message, the simulation applications using the PIM algorithm run more efficiently than applications employing the FIM algorithm and the STIM algorithm. Therefore, in our experiments, the speedup of the PIM algorithm (about 4.12 for comparing 10 federates to 2 federates) is higher than that of the FIM algorithm (about 2.31) and the STIM algorithm (about 3.49). The analysis of effectiveness is based on the results of experiments in this study.

\section{Conclusions and future works}

Interest matching is a very important data filtering mechanism for large-scale distributed simulations and DVE. A good IM algorithm can substantially reduce the transmission of irrelative messages among virtual entities and enhance the scalability of DVE. Most of the IM algorithms in the literature perform overlap tests of regions at discrete time intervals. To capture the events occurring between two consecutive timesteps, they usually use the frequent matching algorithm and the divide-and-conquer method. Additionally, they do not use the motion function of virtual entities for the computation of region overlaps. As a result, they cannot accurately detect all events between two consecutive timesteps or can only decrease the precision of algorithms to report many "spurious events". Moreover, the overhead introduced by redundant matching tests in these algorithms hampers their runtime efficiency.

We present an accurate IM algorithm based on the prediction of the space-time intersection of regions in this study. Our algorithm relies on the motion function of virtual entities to accurately predict the time intervals of overlaps of regions associated with the entities. Based on the 
prediction of the space-time intersection of regions, our algorithm can capture all missing events and does not report the "spurious events". Furthermore, to avoid redundant overlap tests, we use the concept of region pruning to cull out the region pairs that are unlikely to overlap with each other.

We have conducted experiments to compare our algorithm with the existing algorithms on HLAIRTI distributed simulation infrastructure. The experimental results have shown that our PIM algorithm can capture all missing events, while the FIM algorithm fails to report some events. Although the STIM algorithm can detect all missing events, the side effect is that it reports many "spurious events". The experimental results have also shown that through region pruning, PIM has a better ability to avoid redundant matching than FIM, STIM and PIM-BF. Most importantly, the results have shown that there is even extra overhead introduced by solving the intersections of region pairs in our algorithm and that it still has a better runtime performance than FIM, STIM and PIM-BF. Therefore, our algorithm outperforms the FIM, STIM and PIM-BF algorithms in terms of event-capturing ability, redundant matching avoidance, runtime efficiency and scalability.

Although our algorithm has high filtering precision and an efficient runtime performance, it can still be improved in some aspects. First, our algorithm relies on the motion function of virtual entities. The degree of polynomial function to describe the motion of a virtual entity must be less than three in our algorithm. In other words, the polynomial function must be the quadratic polynomial function. We need to extend the polynomial function to other format functions to be compatible with more motions of virtual entities in our future work. Second, our algorithm should be evaluated in more applications in our future work.

\section{Acknowledgments}

The authors gratefully acknowledge the financial support of the National Science Foundation of China (Nos. 61473300 and 61403402). A lot of thanks should be given to referees and editors. Their valuable comments greatly improved the quality of the manuscript.

\section{References}

[1] K.L. Morse, M. Zyda, Multicast grouping for data distribution management, Simulation Practice and Theory, 9 (2002) 121-141.

[2] E.S. Liu and G.K. Theodoropoulos, Space-time matching algorithms for interest management in distributed virtual environments, ACM Trans. Model. Comput. Simul. 24(3) (2014), Article 15, 23 pages.

[3] M.D. Petty, K.L. Morse, The computational complexity of the high level architecture data distribution management matching and connecting processes, Simulation Modelling Practice and Theory 12 (2004) 217-237.

[4] C. Raczy, G. Tan and J. Yu, A sort-based DDM matching algorithm for HLA. ACM Transactions on Modeling and Computer Simulation, 15(1) (2005) 14-38.

[5] K. Pan, S.J. Turner, W.T. Cai, and Z.X. Li, A dynamic sort-based DDM matching algorithm for HLA applications, ACM Transactions on Modeling and Computer Simulation, 21(3) 2011, Article 17.

[6] E.S. Liu, G.K. Theodoropoulos, A fast parallel matching algorithm for continuous interest management, In: Proceedings of the Winter Simulation Conference, 2010, 1490-1590.

[7] H. Abrams, K. Watsen, and M. Zyda, Three-tiered interest management for large-scale virtual 
environments. In: Proceedings of the ACM Symposium on Virtual Reality Software and Technology (VRST), 1998, 125-129.

[8] A. Boukerche and C. Dzermajko, Performance comparison of data distribution management strategies, In: Proceedings of the 5th IEEE International Workshop on Distributed Simulation and Real-Time Applications (DS-RT'01). IEEE Computer Society, Washington, DC, 2001, 67.

[9] G. Morgan and F. Lu, Predictive interest management: An approach to managing message dissemination for distributed virtual environments, In: Proceedings of the 1st International Workshop on Interactive Rich Media Content Production: Architectures, Technologies, Applications, Tools. 2003.

[10] G. Morgan, K. Storey, and F. Lu, Expanding spheres: A collision detection algorithm for interest management in networked games, In: Proceedings of the 3rd International Conference on Entertainment Computing (ICEC'04), 2004.

[11] K. Pan, X.Y. Tang, and W.T. Cai, Hierarchical interest management for distributed virtual environments. SIGSIM-PADS'13, Montréal, Québec, Canada, 2013, 137-146.

[12] J. Ahn, C. Sung, and T. G. Kim. A binary partition-based matching algorithm for data distribution management, In: Proceedings of the 2011 Winter Simulation Conference, 2011, 2728-2739.

[13] IEEE. 2000. Standard 1516 (HLA Rules), 1516.1 (Federate Interface Specification), and 1516.2 (Object Model Template).

[14] K.L. Morse and M.D. Petty, Data distribution management migration from DoD 1.3 to IEEE 1516, In: Proceedings of the 5th IEEE International Workshop on Distributed Simulation and Real Time Applications, 2001, 58-65.

[15] K.L. Morse and J.S. Steinman, Data distribution management in the HLA-multidimensional regions and physically correct filtering, In: Proceedings of the Spring Simulation Interoperability Workshop (SIW'97), 1997, 343-352.

[16] D.J. Van Hook, S.J. Rak, and J.O. Calvin, Approaches to relevance filtering, In: Proceedings of the 11th Workshop on Standards for the Interoperability of Distributed Simulations, 1994, 26-30.

[17] D. J. Van Hook and J.O. Calvin, Data distribution management in RTI 1.3, In: Proceedings of the Spring Simulation Interoperability Workshop, 1998, Paper 98S-SIW-206.

[18] D.D. Wood, Implementation of DDM in the MAK high performance RTI, In: Proceedings of the Spring Simulation Interoperability Workshop, 2002, Paper 02S-SIW-056.

[19] S.J. Rak and D.J. Van Hook, Evaluation of grid-based relevance filtering for multicast group assignment, In: Proceedings of the 14th DIS Workshop on Standards for the Interoperability of Distributed Simulations, 1996, 739-747.

[20] C.E. Bezerra, F.R. Cecin, and C.F.R. Geyer, A3: A novel interest management algorithm for distributed simulations of MMOGs, In: Proceedings of the 12th IEEE/ACM International Symposium on Distributed Simulation and Real-Time Applications (DS-RT 2008). IEEE Computer Society, 2008, 35-42.

[21] M.R. Macedonia, M.J. Zyda, D.R. Pratt, D.P. Brutzman, and P.T. Barham, Exploiting reality with multicast groups: A network architecture for large-scale virtual environments, In: Proceedings of the 1995 IEEE Virtual Reality Annual International Symposium (VRAIS'95), 1995, 2-10. 
[22] I. Kazem, D.T. Ahmed, and S. Shirmohammadi, A visibility-driven approach to managing interest in distributed simulations with dynamic load balancing, In: Proceedings of the $11^{\text {th }}$ IEEE International Symposium on Distributed Simulation and Real-Time Applications (DS-RT'07), 2007, 31-38.

[23] Y. Lu, Y. Wang, and H. Liu, interest management architecture by ALM and region partition for large-scale distributed virtual environment. Journal of Computers, 5(6) 2010, 836-843.

[24] C. Greenhalgh and S. Benford, MASSIVE: A collaborative virtual environment for teleconferencing, ACM Transactions on Computer Human Interactions, 2(3) 1995, $239-261$.

[25] A. Boukerche and C. Dzermajko, Performance comparison of data distribution management strategies, In: Proceedings of the 5th IEEE International Workshop on Distributed Simulation and Real-Time Applications (Cincinnati, OH), 2001, 67-75.

[26] G. Tan, R. Ayani, Y. Zhang, and F. Moradi, An Experimental platform for data management in distributed simulation, In: Proceedings of Simulation Technology and Training Conference (Sydney, Australia), 2000, 371-376.

[27] G. Tan, R. Ayani, Y. Zhang, and F. Moradi, Grid-based data management in distributed simulation, In: Proceedings of the 33rd Annual Simulation Symposium (Washington, DC), 2000, 7-13.

[28] R. Ayani, F. Moradi, and G. Tan, Optimizing cell-size in grid-based DDM, In: Proceedings of 14th Workshop on Parallel and Distributed Simulation (Bologna Italy), 2000, 93-100.

[29] G. Tan, Y. Zhang, and R. Ayani, A hybrid approach to data distribution management, In: Proceedings of the 4th IEEE International Workshop on Distributed Simulation and Real-Time Applications, 2000, 55-61.

[30] A. Boukerche, N.J. Mcgraw, and R.B. Araujo, A grid-filtered region-based approach to support synchronization in large-scale distributed interactive virtual environments, In: Proceedings of the International Conference on Parallel Processing Workshops, 2005, 525-530.

[31] S. Prabhakar, Y. Xia, ,D.V. Kalashnikov, W.G. Aref, and S.E. Hambrusch, Query indexing and velocity constrained indexing: scalable techniques for continuous queries on moving objects. IEEE Trans. Comput. 51(10) 2002, 1124-1140.

[32] Y. Tao, D. Papadias, and J. Sun, The TPR*-tree: An optimized spatio-temporal access method for predictive queries, In: Proceedings of the International Conference on Very Large Data Bases (VLDB), 2003, 790-801.

[33] D.V. Kalashnikov, S. Prabhakar, and S.E. Hambrusch, Main memory evaluation of monitoring queries over moving objects. Int. J. Distrib. Parall. Datab, 15(2), 2004, 117-135.

[34] X. Yu, K.Q. Pu, and N. Koudas, Monitoring k-nearest neighbor queries over moving objects, In: Proceedings of the 21st International Conference on Data Engineering (ICDE’05), 2005, 631-642.

[35] J. Yu, C. Raczy, and G. Tan, Evaluation of sort-based matching algorithm for the DDM, In: Proceedings of the 16th Workshop on Parallel and Distributed Simulation, 2002, 68-75.

[36] L. Wang, H. Zhang, K. Li, and H. Ju, Research and implementation of improved sort-based matching algorithm in data distribution management, Computer Engineering and Applications, 43 (33) 2007, 161-163.

[37] M. D. Petty, Comparing high level architecture data distribution management specifications 1.3 and 1516, Simulation Practice and Theory, 9 (3-5) 2002, 95-119. 
[38] D.J. Van Hook, S.J. Rak, J.O. Calvin, Approaches to RTI implementation of HLA data distribution management services, In: Proceedings of the 15th Workshop on Standards for the Interoperability of Distributed Simulations, Orlando, FL, September 16-20 1996, 535-544.

[39] K.L. Morse, An Adaptive, Distributed Algorithm for Interest Management, Ph.D. Dissertation, University of California Irvine, Irvine CA, May 2000.

[40] R.M. Fujimoto, Parallel and Distributed Simulation Systems, John Wiley and Sons. 2000.

[41] J.D. Cohen, M.C. Lin, D. Manocha, and M.K. Ponamgi, I-COLLIDE: An interactive and exact collision detection system for large-scale environments, In: Proceedings of the 1995 Symposium on Interactive 3D Graphics, ACM, 1995, 189-196.

[42] D. Hanawa and T. Yonekura, A proposal of dead reckoning protocol in distributed virtual environment based on the Taylor expansion, In: Proceedings of the 2006 International Conference on Cyberworlds (CW'06), 2006, 107-114.

[43] https://en.wikipedia.org/wiki/Precision_and_recall.

[44] Y. Peng, Y. Cai, R.H. Zhong, K.D. Huang, Parallel framework for HLA federate Oriented to simulation component on multicore platform, journal of software, 23(8), 2012, 2188-2206.

[45] D.T. Maxwell, An overview of The Joint Warfare System (JWARS), Phalanx, 33(2), 2000 12-32.

[46] F.A. Bowers and D.L. Frochnow, JTLS-JCATS federation support of emergency response tralning, In: Proceedings of the 2003 Winter Simulation Confwence, 2003, 1052-1060.

[47] P.J. Warhola, an analysis of alternative methods to conduct high-resolution activities in a variable-resolution simulation, master dissertation, naval postgraduate school, 1997.

[48] S. Singhal and M. Zyda, Networked Virtual Environments: Design and Implementation, 1999, Addison-Wesley.

[49] D.C. Miller, and J.A. Thorpe, SIMNET: The advent of simulator networking, In: Proc. IEEE 83(8) 1995, 1114-1123.

[50] L. Pantel and L.C. Wolf, On the suitability of dead reckoning schemes for games, In: Proceedings of the 1st Workshop on Network and System Support for Games (NetGames '02), ACM, New York, NY, 2002, 79-84.

[51] Z. Li, X. Tang, W.T. Cai, and S. J. Turner, Fair and Efficient Dead Reckoning-Based Update Dissemination for Distributed Virtual Environments, 2012 ACM/IEEE/SCS 26th Workshop on Principles of Advanced and Distributed Simulation, 2012, 13-22.

[52] A. Mccoy, D. Delaney, S. Mcloone, and T. Ward, Dynamic hybrid strategy models for networked multiplayer games, In: Proceedings of the 19th European Conference on Modelling and Simulation (ECMS '05), 2005, 727-732.

[53] X. Zhang, T. Ward, and, S. McLoone, Comparison of predictive contract mechanisms from an information theory perspective, ACM Trans. Multimedia Comput. Commun. Appl. 8(2), Article 18, 2012, 18 pages.

[54] P.A. Fishwickm and B.P. Zeigler, A Multi-model Methodology for Qualitative Model Engineering, ACM Transactions on Modeling and Computer Simulation (TOMACS), 1992, 2(1): 52-81.

[55] A. Tolk, Engineering Principles of Combat Modeling and Distributed Simulation, Hoboken, NJ: John Wiley and Sons, Inc. 2012. 
[56] H. Vangheluwe, J. de Lara, and P.J. Mosterman, An Introduction to Multi-paradigm Modeling and Simulation, In: Proceedings of the AIS'2002 Conference (AL Simulation and Planning in High Autonomy Systems), Lisboa, Portugal, 2002, 9-20.

[57] P.J Mosterman, An Overview of Hybrid Simulation Phenomena and their Support by Simulation Packages, In: Proceedings of the Second International Workshop of Hybrid Systems: Computation and Control (Lecture Notes in Computer Science 1569). New York, NY: Springer, 1999, 165-177.

[58] A. Borshchev, Multi-method Modeling, In: Proceedings of the 2013 Winter Simulation Conference: Simulation: Making Decisions in a Complex World. Piscataway, NJ: IEEE, 2013, 4089-4100.

[59] A. Sulistio, C.S. Yeo, and R. Buyya, A Taxonomy of Computer-based Simulations and its Mapping to Parallel and Distributed Systems Simulation Tools, Software: Practice and Experience, 34(7) 2004, 653-673.

[60] J. Lynch, S. Y. Diallo, A taxonomy for classifying terminologies that describe simulations with multiple models, In: Proceedings of the 2015 Winter Simulation Conference, 2015, 1621-1632.

[61] E. Cayirci, Multi-resolution federations in support of operational and higher level combined/joint computer assisted exercises, In: Proceedings of the 2009 Winter Simulation Conference, 2009, 1787-1797.

[62] G.A. Plotz, Dr. J. Prince, Multi-Resolution Modeling with a JMASS-JWARS High Level Architecture (HLA) Federation, In: Proceedings of SPIE Enabling Technologies for Simulation Science VII, Vol. 5091, 2003, 350-356.

[63] R. Radhakrishnan and P.A. Wilsey, Ruminations on the Implications of Multi-Resolution Modeling on DIS/HLA, In: Proceedings 3nd IEEE workshop on Distributed Interactive Simulation and Real-Time Applications, 1999, 101-108.

[64] F.A. Bowers, D.L. Prochnow, JTLS-JCATS federation support of emergency response training, In: Proceedings of $35^{\text {th }}$ Winter Simulation Conference, 2003, 1052-1060.

[65] G. Tan, W.N. Ng, and F. Moradi, Aggregation/Disaggregation in HLA Multi-Resolution Distributed Simulation, In: Proceedings 5th IEEE workshop on Distributed Interactive Simulation and Real-Time Applications, 2001, 76-83.

[66] A. Mccoy, T. Ward, S. Mcloone, and D. Delaney, Multistep-ahead neural-network predictors for network traffic reduction in distributed interactive applications, ACM Trans. Model. Comput. Simul. 17(4), 2007, 16 pages.

[67] Y. Yu, Z. Li, L. Shi, Y.C. Chen, and H. Xu, Network-aware state update for large scale mobile games, In: Proceedings of 16th International Conference on Computer Communications and Networks, 2007, 563-568. 